\title{
Finite-volume goal-oriented mesh adaptation for aerodynamics using functional derivative with respect to nodal coordinates.
}

\author{
Giovanni Todarello ${ }^{1}$, Floris Vonck ${ }^{1}$, Sébastien Bourasseau ${ }^{2}$, Jacques Peter ${ }^{1, *}$, \\ Jean-Antoine Désidéri ${ }^{3}$
}

\author{
1 ONERA/DSNA. 29, av. de la Division Leclerc, 92322 Chatillon Cedex, France \\ 2 SNECMA. Rond-Point René Ravaud, 77550 Réau, France \\ ${ }^{3}$ INRIA Sophia Antipolis-Méditerranée. 2004, route des Lucioles - BP 93 06902 Sophia Antipolis \\ Cedex, France \\ *Author for correspondance jacques.peter@onera.fr
}

\begin{abstract}
A new goal-oriented mesh adaptation method for finite volume/finite difference schemes is extended from the structured mesh framework to a more suitable setting for adaptation of unstructured meshes. The method is based on the total derivative of the goal with respect to volume mesh nodes that is computable after the solution of the goal discrete adjoint equation. The asymptotic behavior of this derivative is assessed on regularly refined unstructured meshes. A local refinement criterion is derived from the requirement of limiting the first order change in the goal that an admissible node displacement may cause. Mesh adaptations are then carried out for classical test cases of 2D Euler flows. Efficiency and local density of the adapted meshes are presented. They are compared with those obtained with a more classical mesh adaptation method in the framework of finite volume/finite difference schemes [47]. Results are very close although the present method only makes usage of the current grid.
\end{abstract}

Keywords - Goal oriented mesh adaptation, Discrete adjoint method, Unstructured meshes, Steady compressible equations, Finite-volume scheme

\section{Introduction}

A great asset of unstructured mesh computational fluid dynamics is the possibility to refine the mesh and increase the accuracy of the solution by local mesh adaptation.

\subsection{Feature-based and error-based mesh adaptation}

An intuitive procedure consists in adding nodes where important flow features - shock-waves, vortices, boundary-layers, wakes - are located. To accomplish this, explicit criteria like first or second derivative of a relevant flow variable possibly multiplied by the characteristic local mesh size can be used to control the mesh size $[35,36,3,25,4]$; this technique is called feature-based mesh refinement. A more sophisticated approach consists in deriving a local error estimator to control the adaptation. This can be done according to the residual of the scheme when interpolating the flow onto a finer or coarser grid [22, 23]. Alternately, Frey and Alauzet have used the local interpolation error [15]. They have obtained accurate solutions to transonic flows over refined meshes appropriately adapted in shock regions. Boussetta et al. have proposed a fully-automatic 3D adaptive remeshing procedure based on a Zienkiewicz-Zhu type error estimator and applied it to unsteady (incompressible-flow) metal-forming simulations [6]. Coupez, in a finite-element framework, constructed a node-based metric and established an error estimator per edge [8]. Lastly, let us mention error estimators derived for mesh adaptation in both time and space, as in [16] and [29]. These approaches are based on intuitive or theoretical developments and have revealed to be very efficient in certain contexts.

However, these methods sometimes fail to provide an accurate estimation of the forces or to position satisfactorily the features of interest as well as methods based on regular very fine meshes do. One cause for this inadequacy resides in the fact that the region upstream the features or support of the functions of interest are insufficiently refined [20]. In other words, they may fail to capture propagation effects. A classical example of such problem has been given by Warren et al. [49]. 


\subsection{Output-based mesh adaptation. Proposed method}

In response to this issue, an adjoint-based method can be considered. This is relevant in particular in engineering applications when the accurate prediction of forces and moments is more important than flow-field accuracy all over the fluid domain itself. The adjoint method was first introduced in the field of computational fluid dynamics for shape optimization [18]. It also provides a powerful tool for the error estimation of the output functional(s) of interest since the adjoint equation relates the local error in the field variables to the error in the output functional. Finally, since the Jacobian of convective fluxes appears in this equation, the refinement strategies based on it do refine the zone upstream the output functional support, curing the issue mentionned above.

A clear and detailed state of the art on output-based error estimation and mesh adaptation has been recently published by Fidkowski and Darmofal [20]. Their article covers both finite-element/discontinuous Galerkin and finite volume method. Although our study is intended to apply in the context of finitevolume approximations, we would like to mention that goal-oriented error-based adaptation has achieved a notable degree of performance in simulations by Discontinuous Galerkin (DG) schemes. See for example the contributions of Hartmann et al and their bibliography for simulation of compressible Euler equations [26], laminar Navier-Stokes equations [27] and Reynolds-Averaged Navier-Stokes (RANS) in k- $\omega$ formulation [28]. In particular, the last reference documents a convincing goal-oriented mesh-refinement demonstration for a wing-fuselage configuration by schemes of order 2,3 and 4 .

The proposed method is based on goal sensitivity to allowable node displacements and has been assessed for finite-volume schemes. It is now introduced in the context output-based mesh refinement for this class of schemes.

Until now, the Venditti and Darmofal method is a major reference for goal oriented mesh refinement in the context of finite-volume schemes [46, 47]. It can be seen as an extension of the method of Pierce and Giles [37, 38] sharing the same expressions for a linear functional but with simpler expressions in the nonlinear case. Two grid levels are considered: a coarse grid where all calculations (either direct or adjoint) are tractable and a fine grid where direct and adjoint simulations are considered prohibitively expensive with the exception of a single explicit stage evaluation. The dominant error term in the goal (denoted $J$ from now on) estimated on the coarse grid appears (from Taylor expansions and discrete adjoint equation) to be the residual of coarse grid flow-field interpolated onto the fine grid scaled by the fine-grid adjoint corresponding to this flow approximation. This expression is rewritten to remove the (presumably too expensive to calculate) fine-grid adjoint, replacing it by the coarse-grid adjoint interpolated onto the fine grid. This leads to two terms: a term correcting to the evaluation of $J$ called "computable correction" and an "error in computable correction" providing a criterion for mesh adaptation. This method is based on a rigorous estimation of the error in the functional and has been applied by many authors (see references listed in the introduction of [30]). It has unfortunately the drawback to require two grid levels and raises associated technical issues (in particular fine grid residual evaluation coded if possible without full interpolated flow and fine grid storage).

The search for a criterion using the adjoint vector on a unique grid level is rarely considered in the literature. However the contribution of Dwight [13, 14] must be noted. His method only requires one grid level but it is limited to the classical Jameson et al. numerical scheme [17]. Dwight considered classical test cases for Euler flows. In his work, computations have been performed using Jameson et al. scheme [17] on hierarchies of grids and for different sets of artificial dissipation coefficients $\left(k^{2}, k^{4}\right)$. The error in the functions of interest appeared to be mainly due to artificial dissipation. On this basis, a measure for the approximation error in $J$ for the Jameson et al. scheme is proposed based on the derivatives of $J$ w.r.t. $k^{2}$ and $k^{4}$. Then considering that the dissipation coefficients could vary independently for all faces, a local form of the error due to first and third order-difference dissipative fluxes has been derived and successfully used as the criterion for goal-oriented local mesh refinement.

Recently Fidkowski and Roe have proved for Euler flows that the vector of entropy variables (derivatives of entropy w.r.t. conservative variables), is the adjoint vector of the net entropy production in the computational domain; this property has been then extended to viscous flows [19]. The classical adjointbased error estimation for this specific functional can hence be used without solving an adjoint equation. Fidkowski and Roe have then presented mesh adaptations with a discontinuous Galerkin scheme [26]. In a later article, the authors have pointed out the link between net entropy production and drag in the framework of far-field drag analysis and they extended their method to drag error estimation and mesh adaptation in two dimensions [21].

The purpose of this work is to study a heuristic mesh refinement method based on node displacement sensitivity, that does not require a finer grid and that is not attached to a specific scheme or function of interest. This refinement criterion was already demonstrated in the context of structured meshes 
$[30,32]$. Not only are these type of meshes far less favourable to mesh adaptation and refinement than unstructured meshes, but the classical geometric dependencies of the considered fluxes are not the same for structured and unstructured meshes and a renewed study is required.

\subsection{Isotropic versus anisotropic output-based mesh adaptation}

The proposed method and the previously quoted contributions to the field of finite-volume output-based refinement perform isotropic adaptation that is generally satisfactory for Euler flow simulations. For the sake of completeness, we briefly mention here few authors who derived anisotropic meshes [31, 52] based on a continuous field of Riemannian metrics over the fluid domain. Loseille, Dervieux and Alauzet have analyzed the relationship between anisotropic unstructured mesh-adaptation and goal-oriented error estimate [31]. This link has permitted them to define an output-oriented estimate involving the interpolation error. They have solved formally the mesh optimization formulation for the local metric and adapted subsequently the mesh. They have finally presented adaptations for configurations with complex sets of shock-waves or shock-waves propagating from an aircraft to the ground where anisotropic adaptation is fruitful. Yano and Darmofal [52] have later introduced a Riemannian metric optimization framework for anisotropic simplex mesh adaptation in the context of DG schemes. They have used local solves to estimate the gradient in the metric space of the output-based local error function before performing gradient descent to drive the new mesh towards optimality. They have provided demonstrations for canonical problems and for the convection diffusion equation.

\subsection{Outline}

In Section 2, the expression of the total derivative of an aerodynamic function w.r.t. volume mesh coordinates and the usage of this vector field for shape optimization and mesh adaptation are recalled. In Section 3 the mesh adaptation procedure is briefly described. Section 4 is then devoted to the numerical choices retained. Finally section 5 provides a precise comparison of Venditti and Darmofal method and the present one. Aspects of adjoint and flow solutions, convergence towards limiting function values, are discussed in four test cases presented in former classical articles. Conclusions are drawn in the last section.

\section{Total derivative of functional output w.r.t. volume mesh coor- dinates}

Let us define the basic notations employed here for finite-volume CFD computations: $W$ is the flow field ( $\left.\operatorname{size} n_{W}\right), X$ is the vector of volume mesh coordinates (size $n_{X}$ ) and $R$ is the residual of the scheme. At steady state, these variables satisfy $R(W, X)=0$ (set of $n_{W}$ non-linear equations to be solved for $W$ ). The residual $R$ is supposed to have $C^{1}$ regularity w.r.t. its two vector arguments. At the neighbourhood of $\left(W_{i}, X_{i}\right)$ such that $R\left(W_{i}, X_{i}\right)=0 \operatorname{det}\left[(\partial R / \partial W)\left(W_{i}, X_{i}\right)\right] \neq 0$, the implicit functions theorem allows us to express $W$ as a function of the mesh $X$. This is assumed to be true for all the cases of interest.

\section{$2.1 d J / d X$ for sensitivity analysis and shape optimisation}

In this framework detailed above an aerodynamic function $\mathrm{J}(W, X)$ can be expressed as a function $J$ of the mesh only $J(X)=\mathrm{J}(W(X), X)$, whose derivative w.r.t. $X$ is called here the total derivative of the functional output w.r.t. volume mesh coordinates. It naturally appears in the equation of sensitivity computation for shape optimization using the adjoint vector method: denoting $\alpha$ the vector of design parameters $\left(\right.$ size $n_{\alpha}$ ) and $\mathcal{J}$ the function of interest expressed as a function of the design parameters $(\mathcal{J}(\alpha)=\mathrm{J}(W(X(\alpha)), X(\alpha)))$, the classical equations of the adjoint vector method are:

$$
\left(\frac{\partial R}{\partial W}\right)^{T} \Lambda=-\left(\frac{\partial \mathrm{J}}{\partial W}\right)^{T} \quad \frac{d \mathcal{J}}{d \alpha_{l}}=\frac{\partial \mathrm{J}}{\partial X} \frac{d X}{d \alpha_{l}}+\Lambda^{T}\left(\frac{\partial R}{\partial X} \frac{d X}{d \alpha_{l}}\right) \quad l \in\left[1, n_{\alpha}\right]
$$

or

$$
\frac{d \mathcal{J}}{d \alpha_{l}}=\left[\frac{\partial \mathrm{J}}{\partial X}+\Lambda^{T} \frac{\partial R}{\partial X}\right] \frac{d X}{d \alpha_{l}} \quad l \in\left[1, n_{\alpha}\right]
$$

This clearly identifies

$$
\frac{d J}{d X}=\frac{\partial J}{\partial X}+\Lambda^{T} \frac{\partial R}{\partial X}
$$


This quantity was first introduced by Nielsen and Park in the framework of adjoint based sensitivity analysis for shape optimization [34]. In this context the CPU time needed for the evaluations of the derivatives of the output of interest was (almost) independent of the number of design parameters but, on the contrary, the memory requirements were proportional to the number of design parameters. Actually the storage of volume mesh sensitivity w.r.t. design variables was not possible for large configurations and a few hundreds design parameters and this limited the benefit of the adjoint method. Nielsen and Park proposed an elegant solution involving the adjoint of the explicit or implicit relationship between the deformation of the surface mesh - denoted $S$ - and volume mesh deformation. The equations of the method are presented in [34] and recalled in [32].

It is important to note that $d J / d X$ is an output (and, in some cases, the standard output) of the adjoint module of several large CFD codes $[7,50]$. This allows to make the CFD code independent of the shape parametrization and mesh deformation processes that is a significant asset compared to older practices when the adjoint modules directly calculated the output sensitivities w.r.t. design parameters.

It is finally useful to rewrite $d J / d X$ in the case of a very simple model and scheme to have differences of $\Lambda$ appear in equation (2) ; this will later help understanding the zones of high mesh density obtained with the proposed adaptation method (section §5.4). In case $R$ is the balance of only one numerical flux $F$ and $F$ only involves the local surface vector and the two neighboring states of the face, we denote $i$ the index of an interior node, $A(i)$ the indices of all faces including node $i, l(f)$ and $r(f)$ the indices of the left and right cells of face $f$. The total derivative $d J / d X_{i}$ can then also be expressed as

$$
\frac{d J}{d X_{i}}=\frac{\partial J}{\partial X_{i}}+\sum_{f \in A(i)}\left(\Lambda_{l(f)}-\Lambda_{r(f)}\right) \frac{\partial F^{f}}{\partial X_{i}} .
$$

\section{$2.2 d J / d X$ for $J$-oriented mesh adaptation}

Although not related to mesh refinement but to mesh deformation, $d J / d X$ may provide valuable information about the suitability of mesh $X$ for the estimation of $J$. The recent work of Diskin, Yamaleev et al. is first recalled [51],[10]: the sensitivity of an exact or approximate functional error w.r.t. volume mesh points is calculated and then diminished by a descent method.

Besides, based on this vector field, several mesh adaptation exercises and methods for structured meshes have been evaluated $[30,32]$ in situations where the limiting function value was not known. A relevant point of view appeared to be the following: A node $X_{i}$ with a large sensitivity $d J / d X_{i}$ and distant neighbouring nodes may cause a large change in $J$ by its isolated move in the polygon defined by the neighbouring nodes according to

$$
J\left(X+d X_{i}\right) \simeq J(X)+\frac{d J}{d X_{i}} d X_{i}
$$

(this, obviously, is subject to the domain of displacement $d X_{i}$ where Taylor expansion (4) is valid; it is checked in section (\$5.1) and found to provide a satisfactory estimation). This denotes an unsound local sensitivity to the position of $X_{i}$ and the corresponding refinement criterion

$$
\left\|\frac{d J}{d X_{i}}\right\| \times l_{i}, \quad l_{i} \text { characteristic local mesh size about } X_{i},
$$

appeared to lead to efficient mesh adaptations [32]. More precisely the considered functions of interest, for the former and present studies are line integrals in 2D and surface integrals in 3D. The involved vector field, denoted $\mathcal{P}(d J / d X)$, is then derived from $d J / d X$ by removing all components that would change the shape of the solid object or the support of the function of interest if distinct. The need for the projection operation $\mathcal{P}$ is briefly discussed in section 5.2 where, for one test case, it is removed from the proposed procedure. It clearly appears that the method then adds too many nodes close to the wall and becomes inefficient.

In [32] a spatial mean of $d J / d X$ was also considered. It did not appear to improve the results for unstructured meshes where true local refinement is possible.

\subsection{Asymptotic behaviour of $d J / d X$ towards dense meshes}

When $J$ is a force estimated by summation over solid walls or through far-field drag breakdown [9] (only calculating line integrals in $2 \mathrm{D}$ and surface integrals in $3 \mathrm{D}$ ) when $R$ is defined as the classical finite-volume 
flux-balance (without division by cell-volume) it is checked that the dimensions of adjoint fields do not involve any length and it is actually observed that the adjoint vector converges towards a limiting field as the mesh is refined.

With these mandatory restrictions for $R$ and $J$, the asymptotic behaviour of $d J / d X$ towards dense meshes was checked for structured meshes, for 2D Euler flows in the case of a second order Roe-MUSCL scheme. In this framework, the partial derivative $\partial \mathrm{J} / \partial X$ is first order in space step whereas the indirect aerodynamic sensitivity $\Lambda^{T}(\partial R / \partial X)$ is second order at interior points (see [30] section 2 and [32] section $2)$.

The current study is devoted to unstructured meshes. The classical MUSCL extrapolation then involves geometrical terms in the definition of the left and right state variables associated to a face contrary to the usual formulas for structured meshes. The asymptotic behaviour of $d J / d X$ is hence to be assessed for this type of mesh. With this goal, different flow regimes about the classical NACA0012 airfoil are considered using a hierarchy of regular meshes built from structured O-meshes [44] by cutting every rectangular cell into two triangular meshes. Three of the resultant meshes are shown in left part of figure 2 . The values of

$$
\mu_{J}=\frac{1}{n_{X}} \sum_{i}\left\|\mathcal{P}\left(d J / d X_{i}\right)\right\|
$$

as a function of $n_{X}$ are then calculated for lift $C L p$ and drag $C D p$ and plotted in figure 2.3 (removing the curve of zero lift calculation of symmetric airfoil at null angle of attack with various symmetric meshes). The approximate minus one slope in log-log scale corresponds to an approximate constant value of $\sum_{i}\left\|\mathcal{P}\left(d J / d X_{i}\right)\right\|$ for the different mesh densities. For this $2 \mathrm{D}$ problem with a fixed domain, this correspond to an average value $\left(\sum_{i}\left\|\mathcal{P}\left(d J / d X_{i}\right)\right\|\right) / n_{X}$ approximately scaling with the square of the characteristic mesh size (more precisely, power fits are done and values lying between 1.7 and 2.3 are obtained for the different flow regimes/functions).

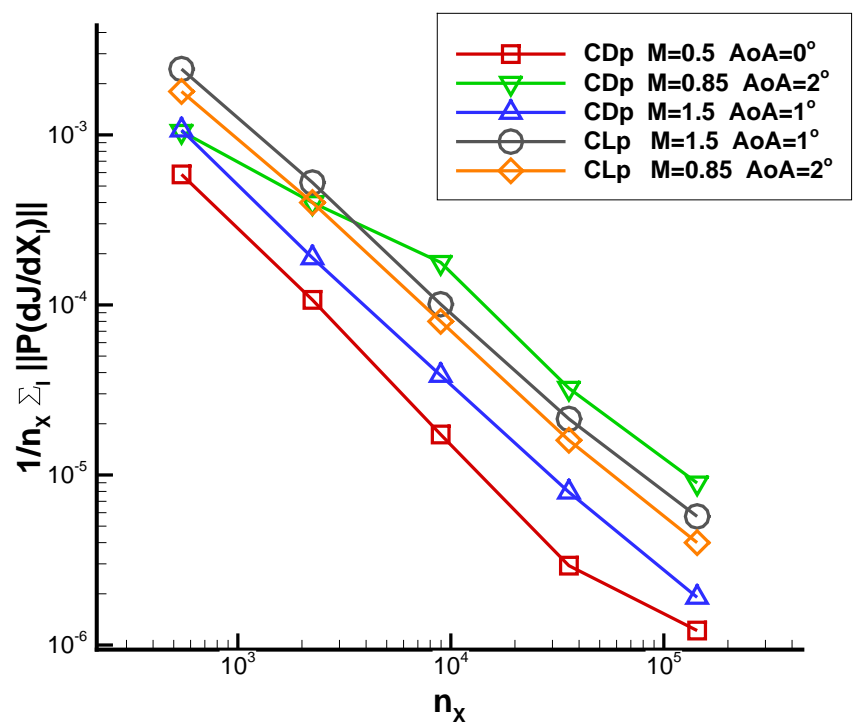

Figure 1: Asymptotic behaviour of $\|d C D p / d X\|\|d C L p / d X\|$ mean over the mesh nodes

It is now discussed whether this global property also corresponds to a stronger local property for the interior nodes. If $d s_{i}$ denotes the area associated to the node of index $i$ in the classical construction of a dual mesh, $\left\|\mathcal{P}\left(d C D p / d X_{i}\right)\right\| / d s_{i}$ and $\|\mathcal{P}(d C L p / d X)\| / d s_{i}$ have been calculated and are plotted for the hierarchy of meshes referenced before (see left column of figure 2). The pattern of isolines of these scalar fields is roughly the same as the mesh refines (see right column of figure 2 for drag). In the next section, an approximate asymptotic behaviour of $\|\mathcal{P}(d J / d X)\|$ will be needed to construct a mesh adaptation strategy; According to the observation above, $\left\|\mathcal{P}\left(d J / d X_{i}\right)\right\|$ will be assumed to scale with the square of the local mesh size $l_{i}$ for $2 \mathrm{D}$ Eulerian flows and for the selected scheme. 

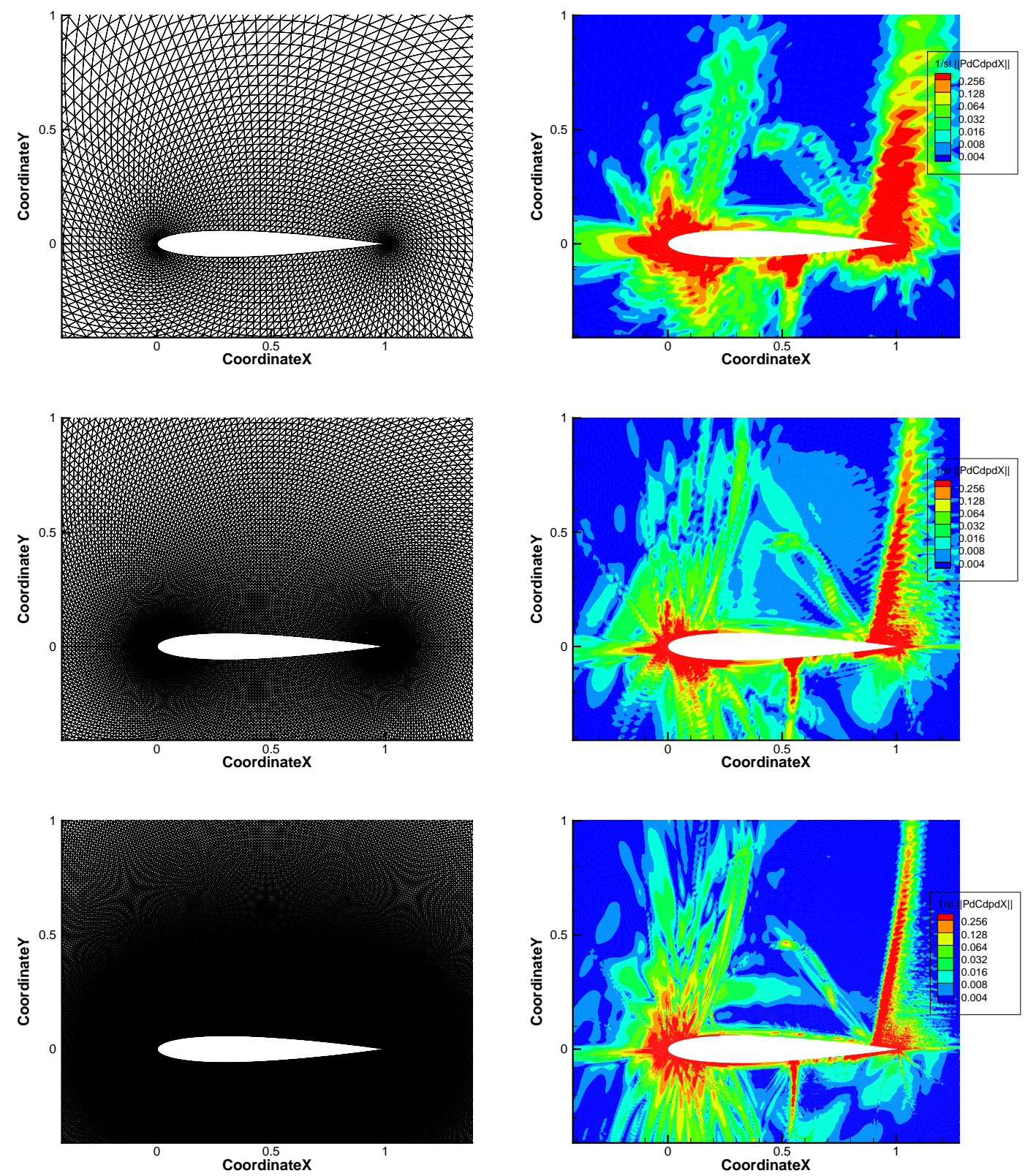

Figure 2: Meshes and corresponding iso-lines of $1 / d s_{i}\left\|\mathcal{P}\left(d C D p / d X_{i}\right)\right\|$ about the NACA0012 $\left(\mathrm{M}_{\infty}=0.85\right.$ AoA $\left.=2^{0}\right)$ 


\section{Adaptation procedure}

As discussed in section (2.2) the proposed goal oriented mesh adaptation method aims at regularizing the first order change that a displacement of a node can cause to the considered output. The retained criterion is

$$
\theta_{i}=\left\|\mathcal{P}\left(\frac{d J}{d X_{i}}\right)\right\| \frac{h_{i}}{2} .
$$

whereby $h_{i}$ is defined as the distance of node $X_{i}$ to the closest neighbouring node.

The corresponding global indicator is

$$
\theta_{J}=\frac{1}{n_{X}} \sum_{i}\left\|\mathcal{P}\left(\frac{d J}{d X_{i}}\right)\right\| \frac{h_{i}}{2}
$$

The adaptation procedure is defined by using a threshold value, $T$, comparing the node criterion values $\theta_{i}$ to the threshold and refining the zones where it is larger. In order to get an approximation of the local change in criterion $\theta$ caused by a local change in characteristic mesh size $h$, it is necessary to make an assumption on the decay of $d J / d X$ when $h$ is decreased: It is simply assumed that the asymptotic behaviour observed for families of meshes in section (2.3) is valid in the case of a local refinement - in other words, that $d J / d X$ decays like $h^{2}$. The new local mesh size $h^{\text {new }}(i)$, where the threshold is exceeded is then

$$
h_{i}^{\text {new }}=h_{i}^{\text {cur }}\left(\frac{T}{\theta_{i}}\right)^{1 / 3} .
$$

If the mesh is adapted by refinement only, the general definition of the aimed characteristic mesh size in

$$
h_{i}^{\text {new }}=h_{i}^{\text {cur }} \max \left(\left(\frac{T}{\theta_{i}}\right)^{1 / 3}, 1\right) .
$$

In accordance to a classical reference on goal oriented mesh refinement [47], the threshold value for $\theta$ will be set in such a way that not more than half the number of nodes are flagged for refinement. The remeshing is performed using MMG2D, from INRIA [11]. This tool uses the classical metric matrix formalism to define the goal shape of the cells in the vicinity of a node. In this case where isotropic mesh refinement is performed, the input provided to MMG2D is a simple diagonal $2 \times 2$ matrix with two equal coefficients equal to the inverse of the square of the desired local mesh size.

\section{Numerical implementation}

The two-dimensional Euler equations are given by:

$$
\frac{d}{d t} \int_{\Omega} \mathcal{W} d S+\oint_{\partial \Omega} \mathcal{F}_{c}(\mathcal{W}) \cdot n d l=0
$$

where $\Omega$ is any fixed surface inside the fluid domain, $\mathcal{W}=(\rho, \rho u, \rho v, \rho E)^{T}$ the conservative variable vector, $n=\left(n_{x}, n_{y}\right)$ the outward normal vector, $\mathcal{F}_{c}$ the convective flux, is (denoting $V=(u, v)$ ):

$$
\mathcal{F}_{c} \cdot n=\left(\begin{array}{c}
\rho V . n \\
\rho u(V . n)+p n_{x} \\
\rho v(V . n)+p n_{y} \\
\rho E(V . n)+p(V . n)
\end{array}\right)
$$

where $p$ stands for the static pressure.

The space domain is discretized by a mesh of triangles only. An extension to unstructured meshes of Roe flux [40] with MUSCL method and van Albada limiting function [1] is retained for the space discretization. The functional outputs of interest are the discretization on the mesh of pressure lift and drag

$$
C D_{p}=\oint_{\Gamma} \frac{2}{\gamma M_{\infty}^{2}}\left(\frac{p}{p_{\infty}}-1\right) \vec{n} \cdot e_{\infty}^{\vec{d}} d l, \quad C L_{p}=\oint_{\Gamma} \frac{2}{\gamma M_{\infty}^{2}}\left(\frac{p}{p_{\infty}}-1\right) \vec{n} \cdot \overrightarrow{f_{\infty}} d l,
$$

where $\vec{n}$ is a local normal vector pointing towards the solid object, $\vec{e}_{\infty}$ is a unit vector aligned with far-field velocity and $\overrightarrow{f_{\infty}}$ is a unit vector such that $\left(\overrightarrow{e_{\infty}}, \overrightarrow{f_{\infty}}\right)$ is direct. 


\section{$5 \quad$ Numerical results}

In order to assess the effectiveness of mesh refinement based on indicator (6)(7) a series of four flows about the NACA0012 airfoil has been considered. The flow conditions cover subsonic to supersonic regimes $\left(\mathrm{M}_{\infty}, \mathrm{AoA}\right)$ equal to $\left(0.5,0^{\circ}\right),\left(0.85,2^{\circ}\right),\left(0.95,0^{\circ}\right),\left(1.5,1^{\circ}\right)$ - and allow comparison of adapted meshes, goal values, efficiency of the adaptation process with former publications [47, 14]. It appeared that drag-oriented mesh adaptation for the subsonic non-lifting test case produces simple meshes with dense zones about and upstream the airfoil. Besides, the comparison of efficiency w.r.t. the classical method introduced by Venditti and Darmofal $[46,47]$ leads to similar analysis than the other cases. For the sake of brevity, a detailed presentation of the more interesting transonic and supersonic cases is preferred. In particular, a very precise analysis of mesh density w.r.t. flow field and adjoint field features is provided for two of the selected cases in section (§5.4).

The classical studies by Venditti and Darmofal [47] and Dwight [14] have shown that specific goaloriented mesh adaptation is more efficient than feature-based adaptation for accurate calculation of functional outputs; they have even confirmed the observation made by Warren et al. [49] that featurebased adaptation may fail to provide correct limiting values for functional outputs. The effectiveness of the current method is hence only compared to the Venditti and Darmofal method $[46,47]$ that has been broadly used and which refinement indicator is directly related to the error in the goal function. This method, considered as a reference, is adapted from cell-vertex framework to the cell-centred framework of the els $A$ code [7] ; the fine 2D meshes are built by splitting triangles in four (method of [47], section $2, N=2$ ). The $T$ parameter of the proposed method and the error bound of the reference method are adjusted at each adaptation step so that the meshes built by the two methods have about the same number of points.

Concerning the limiting function values, they result from a series of calculations on fine structured meshes $[44,45]$ up to $4097 \times 4097$ nodes. Forces are first calculated up to three digits after drag counts $\left(C D p \times 10^{4}\right.$ - also denoted d.c. $)$ and lift counts $\left(C L p \times 10^{2}\right.$ - also denoted l.c.). The classical Richardson extrapolation then provides the limiting values and, among various sets of GCI formulas [39], those presented by Rumsey et al. [42] are used to calculate intervals of confidence about finest grid values. After these preliminary calculations conducted to high accuracy, bounds on goal intervals for function values are rounded off for simplicity and consistency with the desired accuracy. It is finally checked that the series of calculations on the corresponding hierarchy of regular unstructured meshes leads to consistent results.

\subsection{Transonic flow $\mathrm{M}_{\infty}=0.85$ AoA $=2^{0}$}

This test case is a transonic lifting case with a strong shock-wave on the suction side. It is selected to assess the domain of validity of equation (4), to check the need for the projection operator in equation (6) and to perform a lift-oriented mesh adaptation.

The accuracy of calculated output total derivatives w.r.t. mesh coordinates and the validity of equation (4) are checked by independent displacements of nodes and flow calculations performed on a coarse NACA0012 mesh. Six nodes, denoted $X_{k}$ (indicated on figure 3 left), are given 18 displacements $\delta X_{k}$ along the direction of $d C L p / d X_{k}$ (that is also denoted below $\left.n_{k}=\left(d C L p / d X_{k}\right) /\left\|d C L p / d X_{k}\right\|\right)$. The ratio

$$
\frac{(d C L p / d X) \cdot \delta X_{k}-\left(C L p\left(X+\delta X_{k}\right)-C L p(X)\right)}{C L p\left(X+\delta X_{k}\right)-C L p(X)} .
$$

is first computed for the six points $X_{k}$ and it is verified that it does converge towards zero with the node displacement. This proves that the $d C L p / d X$ vector field is correct; more extensive verifications have been reported in [43]. The deviation of $C L p$ with respect to the nominal value,

$$
\varphi\left(\delta X_{k}\right)=C L p\left(X+\delta X_{k}\right)-C L p(X), \quad \delta X_{k}=d /\left(0.5 h_{k}\right) \cdot n_{k},
$$

is then plotted as a function of $d$, the displacement relative to half the local meshes size, $0.5 h_{k}$, in the direction $n_{k}$. The right part of figure 3, first illustrates the much stronger sensitivity of CLp to the coordinates in points $\mathrm{C}$ and $\mathrm{D}$, located close to trailing edge. Besides, it allows to estimate the domain of validity of equation (4) for the selected points: for points A and $\mathrm{E}$, the variation is nearly linear over the interval $d \in[-0.2,+0.2]$; for point $\mathrm{F}$ the domain of linearity would even be larger; at points $\mathrm{C}$ and $\mathrm{D}$, the validity domain is smaller and non symmetric but still represents a significant fraction of the interval; finally at point $\mathrm{B}$ the behavior seems more to be quadratic. 

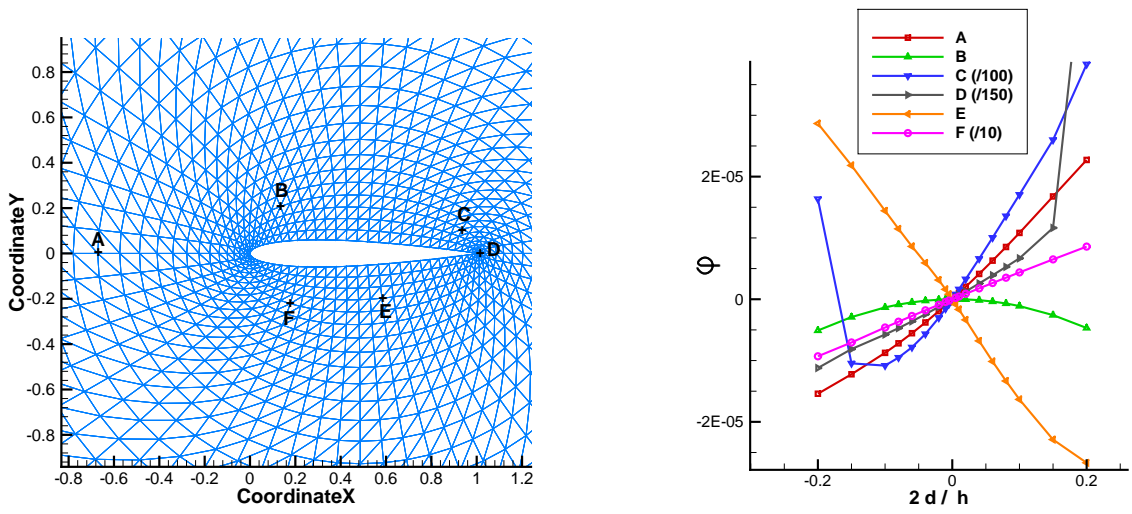

Figure 3: Left: location of points for check of total derivatives of outputs w.r.t. mesh coordinates. Right: evaluation of $C L p$ deviation, $\varphi$, w.r.t. nominal value at points A, B, C, D, E, F (scaled as indicated for points $\mathrm{C}, \mathrm{D}$ and F)

The iso-Mach number lines of the flow obtained on a fine regular grid are presented subsequently in figure 14. A limiting value of 824.152 d.c. and a confidence interval of [823.839,826.963] d.c. based on the finest grid estimation $\left(C D_{1}=825.401 \times 10^{-4}\right)$ are obtained by the procedure detailed in the beginning of the section. Concerning lift, the corresponding quantities are 62.588 l.c. and interval $[62.547,62.952]$ l.c. based on $C L_{1}=0.62750$ and two coarser grid values. Lift-oriented mesh adaptation procedures are run with a 0.001 ( 0.1 l.c.) tolerance w.r.t. the rounded limiting value 62.59 l.c.. As the convergence towards limiting function values is unfortunately quite oscillatory on the available hierarchy of regularly refined structured meshes, only the limiting value is reported on the convergence plots of the case below.

The threshold $T$ - equation (7) - of the proposed method and the error bound of the reference method are adjusted so that the size of the successive adapted meshes is about 3500, 5800, 8800 and 12800 nodes. The lift value on the initial 1352 -node mesh is $61.637 \times 10^{-2}$. It is lowered at the first adaptation step then increased towards the limiting value by the next three adaptation steps (see figure 4 for detailed convergence). Three adaptation steps are required for the $\theta$-indicator method until $C L p$ is included in the goal interval for lift. Three adaptation steps are also needed for the reference method with its correction until CLp estimation is included in the interval $[62.49,62.69]$ l.c. (respectively, five adaptation steps without this correction).

The adapted meshes at step five (fourth adapted meshes) are compared in figure 11 (reference method; 12973 nodes) and 12 (proposed method; 12502 nodes). They actually present very similar dense zones close to the upper side and lower side shock-waves, upwind the profile and along a hat shape above the airfoil. The reason for this will be discussed in section 5.4.

The plots of compared analysis of refinement criteria are figures 22 and 23 of annex B. They exhibit closely similar trends: the two methods initially select for refinement a large area upstream and above the profile. On the second to fifth steps, they alternatively detect a thin area upwind the profile, the upper-side shock-wave and the already mentioned hat shape above the airfoil. When looking closely to pictures 22 and 23, the only clear difference between the error estimator of the reference method and the sensitivity indicator of the proposed method is that the latter one is relatively stronger close to the wall and this leads to a higher mesh density in this zone.

The mean value of criterion $\theta_{i}$ over the mesh, $\theta_{C L p}$, is calculated for all adapted meshes and then plotted in log-log scale as a function of $n_{X}$ - see figure 8 left. It appears to decrease almost linearly in this system of axis with a slope slightly lower than -1.5. A straight-line with -1.5 slope is the expected trend for regularly refined meshes with uniform density $\left(\theta_{J}\right.$ scaling with $1 / n_{X}^{3 / 2}, \mu_{J}$ scaling with $\left.1 / n_{X}\right)$. The stronger decrease of $\theta_{C L p}$ proves the consistency of the refinement procedure and may be related to former results for structured meshes ([30] tables 1,2 and 6)

Finally, the relevance of the projection operation involved in the criterion definition - equation (6) is assessed. As expected from the experience gained with structured meshes, the indicator obtained without the projection operation (denoted no-projection $\theta$-indicator in the legend of figure 4 ) leads to over-refinement close to the wall and stall of $C L p$ value below the limiting value. 


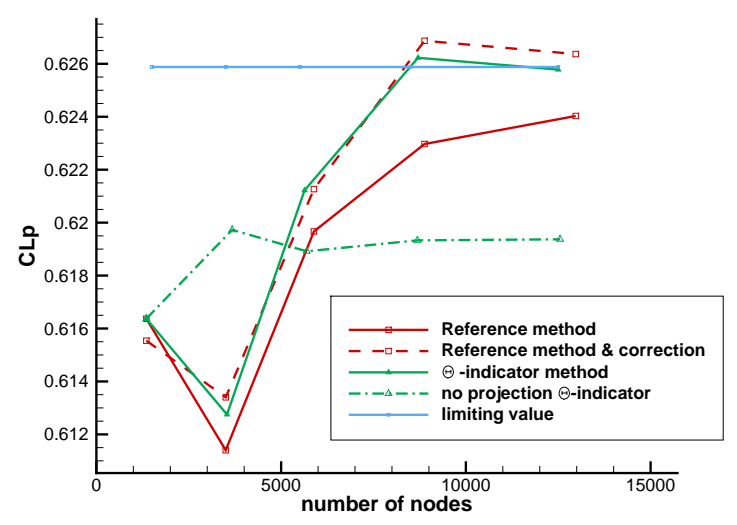

Figure 4: Convergence of $C L_{p}$ for $\theta$-based and reference method $\left(\mathrm{M}_{\infty}=0.85\right.$ and $\left.\mathrm{AoA}=2^{\circ}\right)$

\subsection{Transonic flow $\mathrm{M}_{\infty}=0.95$ AoA $=0^{0}$}

The second test case involves a transonic flow about the NACA0012 airfoil with a free-stream Mach number of $\mathrm{M}_{\infty}=0.95$ and an angle of attack $\mathrm{AoA}=0^{\circ}$. It is selected to allow comparison of flow fields, limiting function value and adapted meshes with those presented in the classical reference [47]. The flow accelerates along the airfoil. It is supersonic from sonic lines (symmetric slightly slanted lines starting at the vicinity of the leading edge) to an oblique fish-tail shock-wave based on the trailing edge. Downstream this first shock-wave, the fluid remains at low supersonic regime. About five chords downstream the airfoil, a weak, almost transverse shock-wave lowers the Mach number to subsonic conditions.

The iso-Mach number lines of the flow obtained on a fine regular grid are presented in figure 5 . The comparison with their counterpart in [47] is very satisfactory. Also very satisfactory is the comparison of limiting drag value: a value of 1098 d.c. is reported on the plots of [47]. A limiting value of 1097.979 d.c. and a confidence interval of $[1097.972,1098.047]$ d.c. based on finest grid estimation $\left(C D_{1}=1098.009 \times\right.$ $10^{-4}$ ) are obtained by the procedure detailed in the beginning of the section.

The $C D p$-oriented mesh adaptation procedure is then run with the higher error tolerance considered in [47] ; This selected 0.001 margin of error is probably standard for relative accuracy of simulation outputs (about 1\% error) and somehow large for drag estimation. Considering this error margin, the goal value is rounded to 0.1098 . The threshold $T$ - equation (7) - of proposed method and the error bound of the reference method are adjusted so that is number of nodes of the successive adapted meshes is about 2800, $4000,5500,7000$.

The drag value on the initial mesh is about 1139 d.c.. It is lowered by the successive adaptation steps. Three adaptation steps are needed for the $\theta$-indicator method until $C D p$ falls in the interval [1088.,1108.] d.c. Four adaptation steps are needed for the reference method until (uncorrected) $C D p$ is included in the interval [1088. , 1108.] d.c. Actually as seen on plot 5 , the drag value of the reference method after three mesh adaptations is quite close to the 1108. d.c. upper bound so that the difference in number of adaptation steps is not very significant. Besides, the drag value including the classical correction term is included in the goal interval after just one adaptation step.

The adapted meshes are compared: Unfortunately, due to the large extent to far-field (150 chords) used in this study, the obtained number of nodes of the adapted meshes cannot be compared with those presented in [47]. Nevertheless, the general aspect of generated meshes can be compared. These adapted meshes obtained by the two considered methods are quite similar - see figure 6 left and right. They exhibit dense mesh zones above, below and upwind the airfoil and have the same general aspect as the mesh presented in [47] figure 7 (a too close comparison being probably impossible due to both, difference in numerical schemes et mesh extension to far-field).

The mean $\theta_{i}$ value over the mesh, $\theta_{C D p}$, is calculated as in previous section for all adapted meshes It is plotted in log-log scale as a function of $n_{X}$ in figure 8 (second left). It appears to decrease irregularly, faster than -2. slope straight line in this frame of reference. This decrease with $n_{X}$ is stronger than the -1.5 (expected for regularly refined meshes with uniform cell size). This is interpreted as previously. 

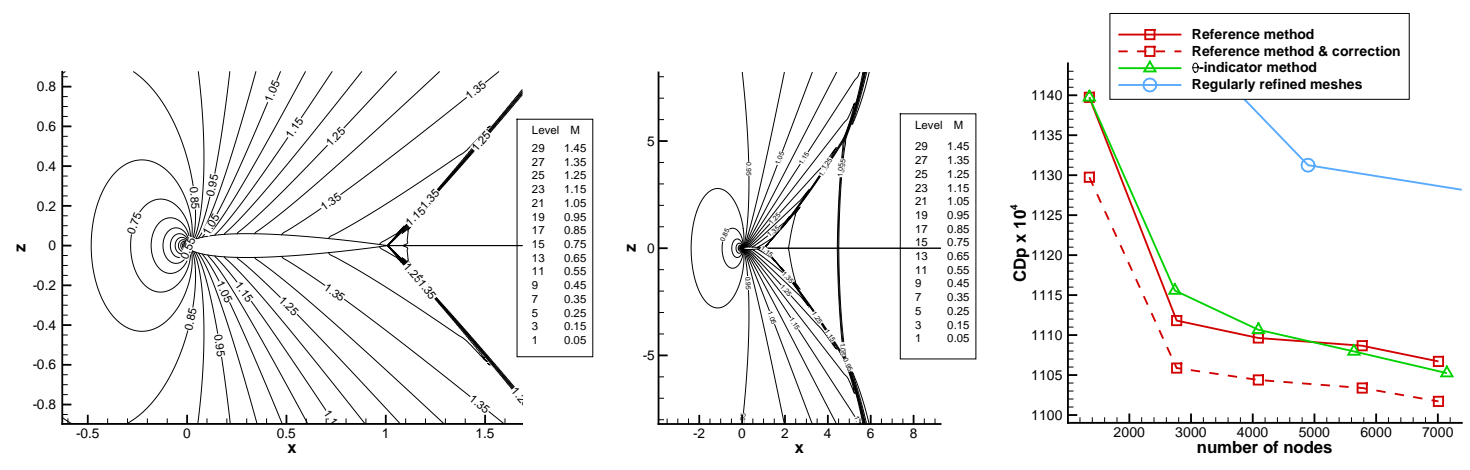

Figure 5: Second transonic case, $\mathrm{M}_{\infty}=0.95, \mathrm{AoA}=0^{\circ}$. Two plots left: Iso-Mach number lines $(\mathrm{M}=$ 0.95 and $\mathrm{AoA}=2^{\circ}$ ). Right: convergence $C D p$-oriented mesh adaptation with 10 drag counts with error tolerance
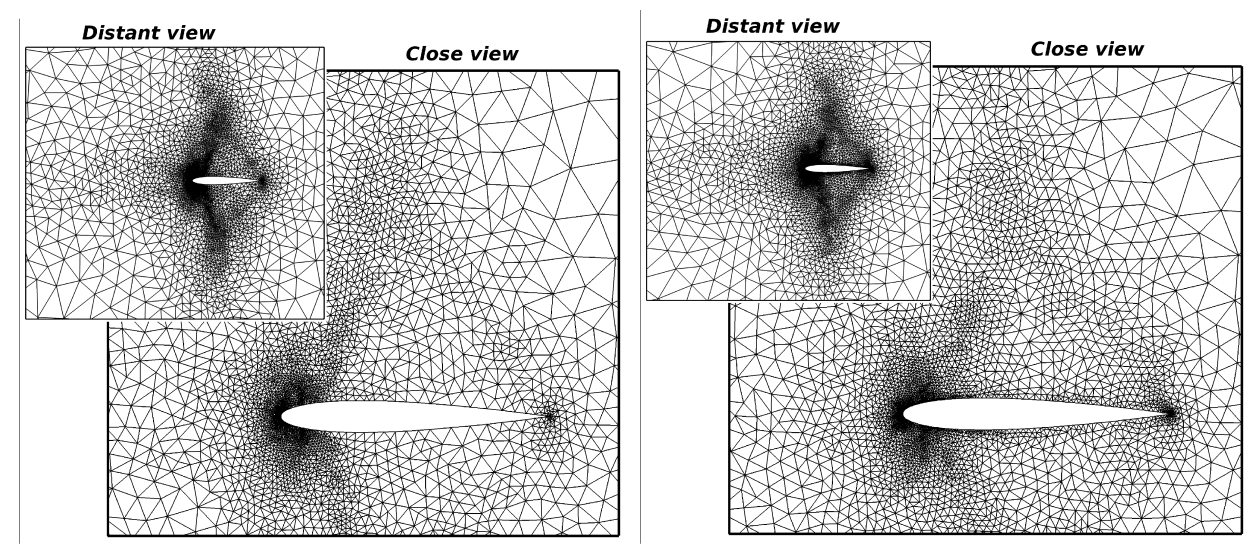

Figure 6: Second transonic case, $\mathrm{M}_{\infty}=0.95, \mathrm{AoA}=0^{\circ}$. Left: adapted meshes obtained by the $\theta$-indicator method at final step. Right: adapted meshes obtained by the reference method at final step

\subsection{Supersonic flow $\mathrm{M}_{\infty}=1.5 \mathrm{AoA}=1^{0}$}

The third test case is a supersonic flow calculation about the NACA0012 airfoil with a free-stream Mach number of $\mathrm{M}_{\infty}=1.50$ and an angle of attack $\mathrm{AoA}=1^{\circ}$. It is selected to allow a comparison of adapted meshes with those presented in the classical reference [14] (that article had indicated differences between the actual functional values and non-displayed limiting function values). According to the theory of supersonic flows about blunt bodies, the flow is supersonic and constant up to a detached shock-wave. Downstream the shock-wave, the flow is subsonic in a small bubble close to the airfoil leading edge and supersonic elsewhere. It accelerates along the airfoil up to a fishtail shock-wave based on the trailing edge. Downstream this second shock-wave the flow is still supersonic with a Mach number close to the upwind far-field Mach number.

A limiting value for lift of 0.0547812 is obtained as well as very small confidence interval [0.0547811, 0.0547816 ] about the finest grid value $C L p_{1}=0.05478$ following [42]. This surprisingly small interval comes from the small differences between lift value for the three finest meshes of the considered hierarchy - $\left(C L p_{1}, C L p_{2}, C L p_{3}\right)=(0.05478,0.05479,0.05479)$ - and the good consistency of the series with Richardson's assumption. Of course this number of significant figures exceeds by far the required accuracy for lift calculation. As lift is quite low for this configuration an goal interval of $+/-0.01$ l.c. is retained for mesh adaptations, requiring lift values in [0.05468, 0.05488].

On the contrary, the three fine grid drag values - $\left(C D p_{1}, C D p_{2}, C D p_{3}\right)=(0.0969133,0.0969039,0.0968980)$ - are not consistent with Richardson's hypothesis since $\left|C D p_{1}-C D p_{2}\right|>\left|C D p_{2}-C D p_{3}\right|$. The limiting value obtained under this hypothesis is hence discarded. Only the finest grid value 0.0969133 and the GCI interval of confidence [0.0969054,0.0969213] are retained. Finally, considering the quite high drag level, a margin of 0.0002 (two d. c.) about the finest grid value is retained for mesh adaptations, requiring 
drag values in the interval with rounded bounds [0.09671, 0.09711]. The threshold $T$ - equation $7-$ of proposed method and the error bound for the reference method are adjusted so that the number of nodes of the successive adapted meshes is about 4000, 6000, 8000, 10000., and then 14000 and 18000 nodes (necessary for drag only).

The $C L p$-oriented mesh adaptation procedure is then applied with the goal interval prescribed above. Only two adaptation steps are needed for the reference method (uncorrected and corrected values) until $C L p$ falls in the interval [0.05468, 0.05488]. Four adaptations steps are required for the proposed method until $C L p$ reaches the goal interval (fourth $C L p$ value obtained after three adaptation steps being very close to the lower bound of the goal interval). Both method are much more efficient than uniform refinement.

The mean of $\theta$ value over the mesh is calculated as in previous section for all adapted meshes and plotted in $\log$ - $\log$ scale versus $n_{X}$ - see figure 8 second right. It appears to be almost linear and the corresponding slope (a bit lower than minus two) leads to the same comment as in section 5.1 and 5.2. Considering the very small uncertainty on the limiting value of lift, the error is calculated and plotted as a function of $\theta_{C L p}$. It appears to be roughly linear in log-log scale - see figure 9. Finally, the error is examined as a function of the total number of points - see figure 10. It is also roughly linear in log-log scale and the regression indicates that it scales with $n_{X}^{-2.82}$. For this mesh adaptation, mainly small triangles are created and the mean characteristic size $\bar{h}$ all over the mesh almost behaves like the inverse of the total number of points. The error hence scales with $\bar{h}^{2.84}$; the exponent exceeding the accuracy of the scheme. The plots of compared analysis of refinement criteria are figures 24 and 25 of annex B. They exhibit surprisingly similar trends: the two methods initially identify a large area about and upstream the profile to be refined. On the second mesh, they roughly detect the specific zone delimited by the detached shock-wave and two sections of characteristic lines between the trailing edge and the shock-wave (The reason why this zone is the zone of main influence for lift evaluation is more precisely discussed in the next section). This specific area is then the only detected zone by the two methods at step three, four and five. When looking closely at the discrepancies between figures 24 and 25, it appears that the error in computable correction is relatively higher close to the wall. This is also confirmed by the densities of the final meshes.

The $C D p$-oriented mesh adaptation procedure is then applied with the goal interval $[0.09671,0.09711]$ previously defined. Seven adaptation steps are needed for both, the reference method (uncorrected value) and the proposed $\theta$-indicator method until $C D p$ is included between the specified bounds. This number is lowered to four for the reference method with correction term.

The mean of $\theta$ over the mesh nodes is also calculated for the drag, as before, for the successive meshes and then plotted in log-log scale versus $n_{X}$ - see figure 8 right. It appears to be almost linear and the corresponding slope (about -2.) is again a sign of consistence of the adapted meshes and the criterion (6) As for lift, just above, considering the small uncertainty on drag limiting value, the error is calculated and plotted as a function of $\theta_{C D p}$. It appears to be almost linear in log-log scale (excluding step two, evidently fortuitously accurate) - see figure 9 Finally, the error is examined as a function of the total number of points - presented figure 10. Excluding the first two steps, it is also roughly linear in log-log scale and the regression indicates that it scales with $n_{X}^{-0.692}$. For this mesh adaptation, as noted for lift previously, the mean characteristic size $\bar{h}$ all over the mesh almost behaves like the inverse of the total number of points. The error hence scales with $\bar{h}^{0.696}$; the exponent being significantly lower than the accuracy of the scheme, contrary to what was observed for lift. 

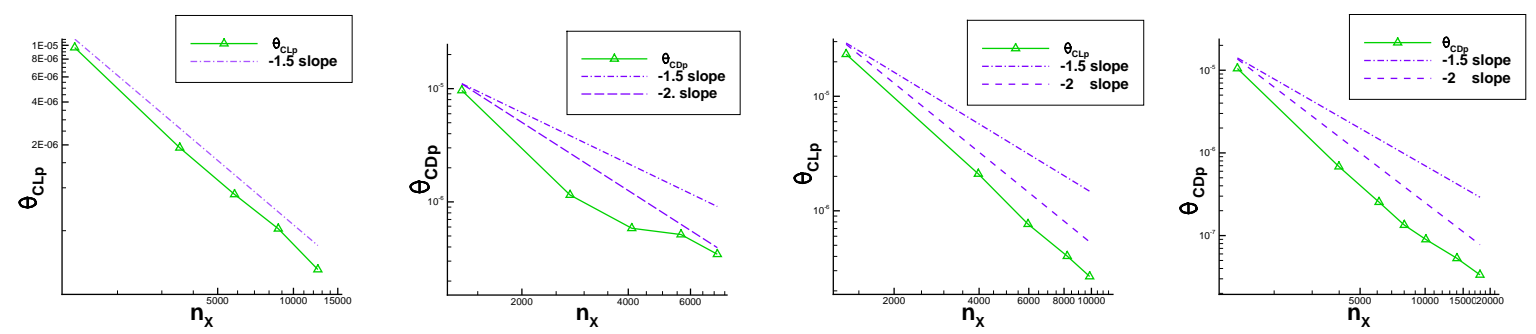

Figure 8: Mean of $\theta$ over the mesh nodes of the successive adapted meshes for (from left to right) (a) $C L_{p}$ first transonic test case $\mathrm{M}_{\infty}=0.85$ and $\mathrm{AoA}=2^{\circ}$ (b) $C D_{p}$ second transonic test case $\mathrm{M}_{\infty}=0.95$ and $\mathrm{AoA}=0^{\circ}$ (c) $C L_{p}$ supersonic test case $\mathrm{M}_{\infty}=1.5$ and $\mathrm{AoA}=1^{\circ}$ (d) $C D_{p}$ supersonic test case $\mathrm{M}_{\infty}$ $=1.5$ and $\mathrm{AoA}=1^{\circ}$
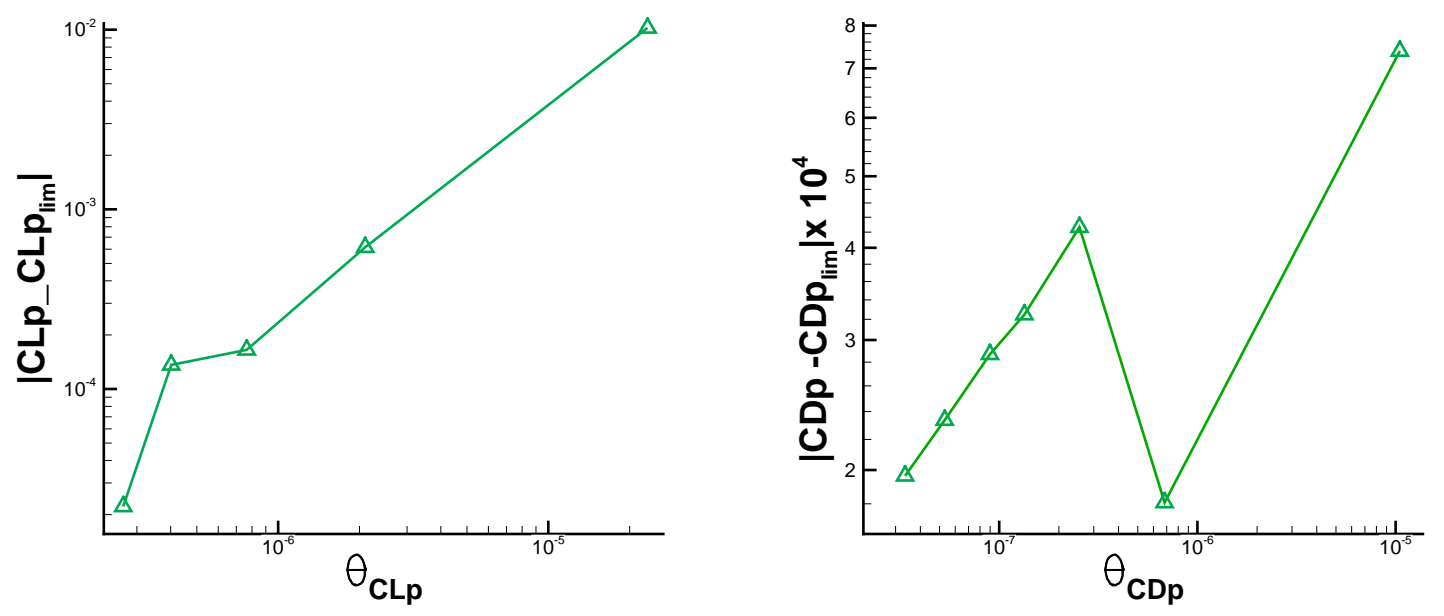

Figure 9: Error on functional output versus mean of $\theta$. Supersonic test case $\mathrm{M}_{\infty}=1.5$ and $\mathrm{AoA}=1^{\circ}$. Left $C L_{p}$, right $C D_{p}$
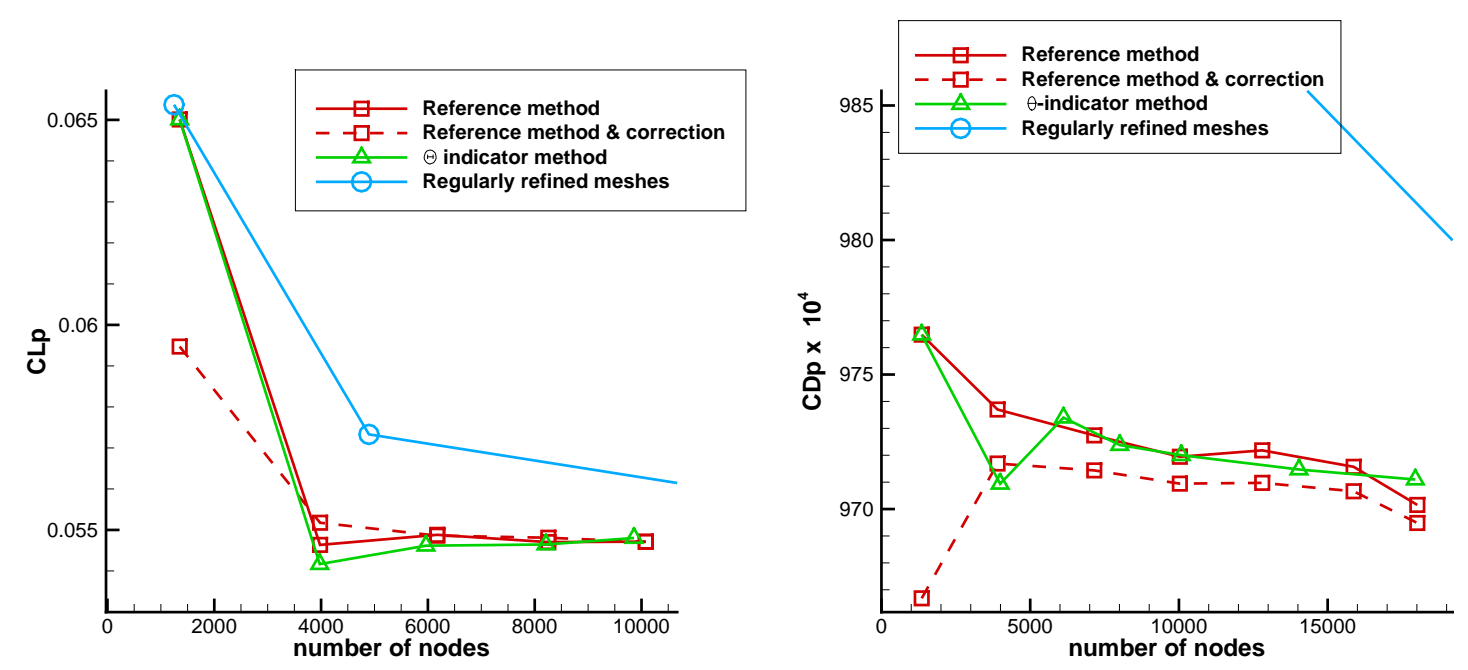

Figure 7: Supersonic test case, $\mathrm{M}_{\infty}=1.5$ and $\mathrm{AoA}=1^{\circ}$. Left: convergence for $C L_{p}$ for $\theta$-based and reference method. Right: convergence for $C D_{p}$ for $\theta$-based and reference method. 

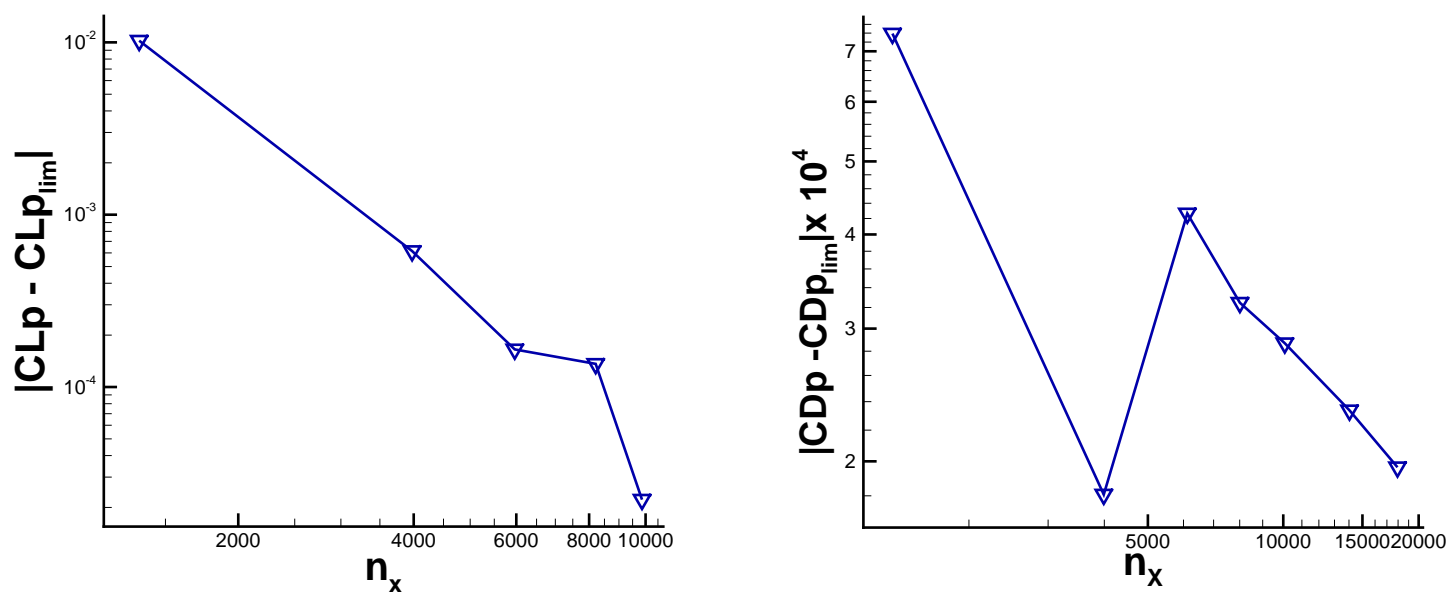

Figure 10: Error on functional output versus total number of points $n_{X}$. Supersonic test case $\mathrm{M}_{\infty}=$ 1.5 and $\mathrm{AoA}=1^{\circ}$. Left $C L_{p}$, right $C D_{p}$

\subsection{Examination of adapted meshes}

The resulting meshes have been examined in all cases. Concerning the non lifting-transonic ( $\$ 5.2)$, the zones refined by the two methods are the vicinity of the profile and the area upwind this zone. A plot is presented in figure 6 The adapted meshes for the two remaining cases - lifting-transonic (\$5.1) and lifting supersonic $(\S 5.3)$ - exhibit more specific mesh density maps with clearer dense and coarse zones that are discussed below.

These meshes are presented for the lift function in figure 11 to 13 (transonic flow) and 18 to 20 (supersonic flow) for the reference method, left ([47]), the proposed $\theta$-based method, in the middle, and Dwight's method (plots copied from [12] [13] or [14] with permission). To help interpreting these density maps, iso-Mach lines and iso-lines of first lift-adjoint component are presented in figure 14 and 21 (the iso-lines of the other lift-adjoint components are quite similar ; the general aspect of the drag adjoint iso-lines is quite similar to the one of lift adjoint). 


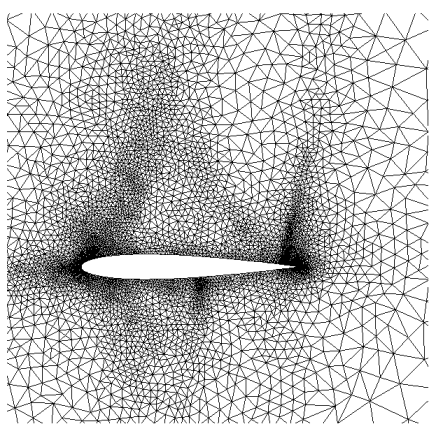

Figure 11: Transonic conditions, Error-based method [47]

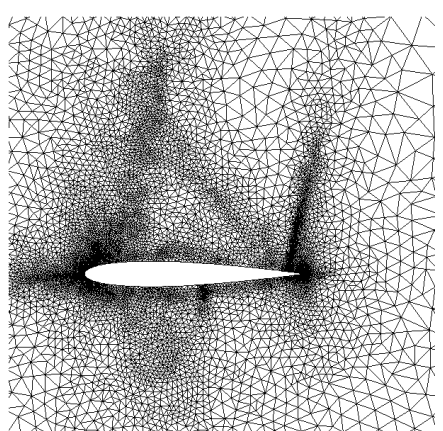

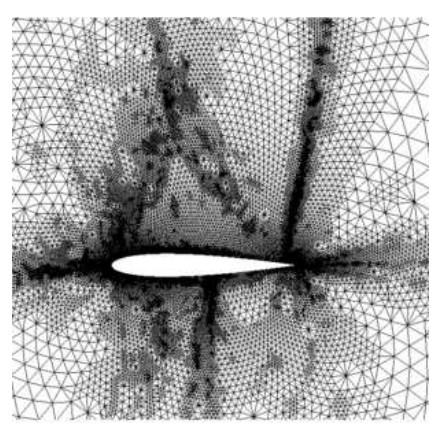

- Figure 13: Transonic conditions, dissipation-based [14]
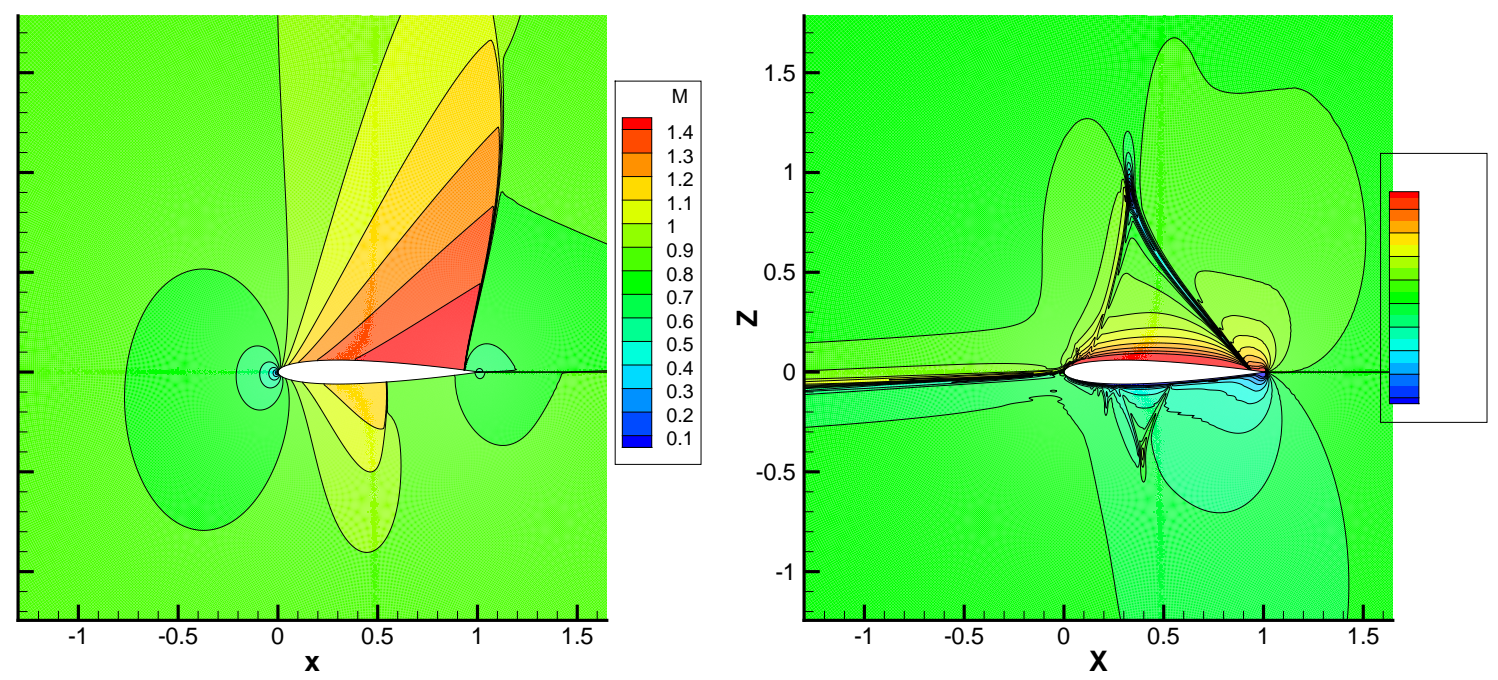

Figure 14: Transonic case $\S 5.1$, iso-Mach number lines $\Delta M=0.1$ (left), iso-lines of first-component of lift adjoint vector (right)

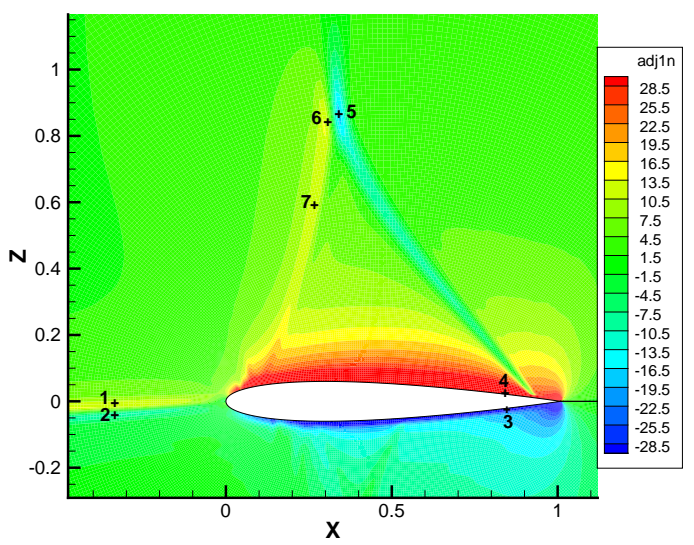

Figure 15: Transonic case $§ 5.1$ position of points for assessment of residual perturbation influence on CLp 


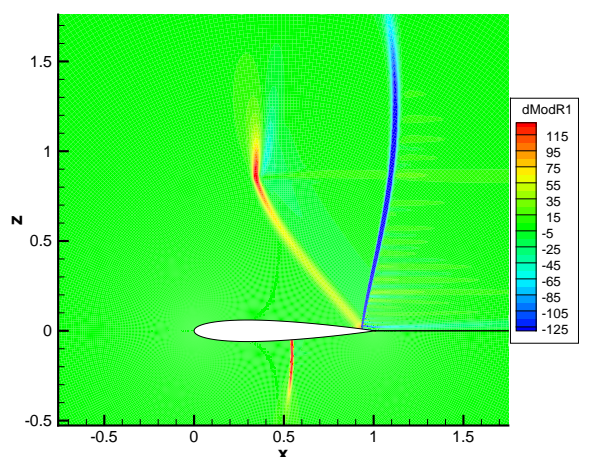

Figure 16: Transonic case $\S 5.1$ change of Mach Figure 17: Transonic case $\S 5.1$ change of Mach number due to change of $R_{1}$ at point 5

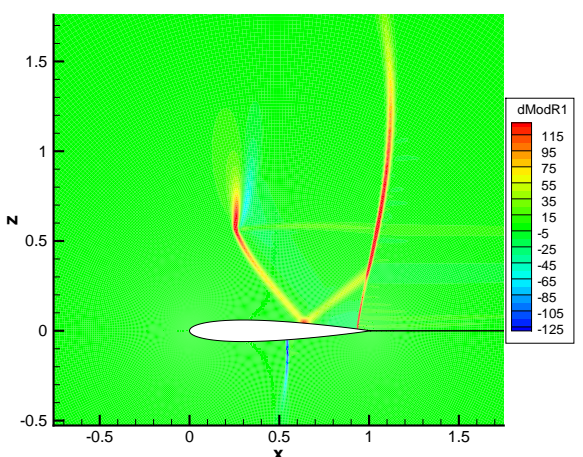

number due to change of $R_{1}$ at point 7

In order to gain insight in the way the adjoint vector vehicles information, recall that this vector is the sensitivity of the function of interest (now specifically the lift coefficient $C L p$ ) to an infinitesimal residual perturbation (see e.g. [20] for a precise explanation). This observation led us to conduct a series of tests in which the (density-related) first component of the residual is perturbed by a small amount $\left(\Delta R_{1}= \pm 2 \times 10^{-4}\right)$, and the resulting variation in the Mach number field is observed. These experiments were made at points 1-7 of Figure 15 which provides the iso-value contours of the corresponding first component of the adjoint vector $\lambda_{1}=\partial C_{z} / \partial R_{1}$. We first check that the sign of $\lambda_{1}$ given by this figure is indeed consistent with the variations in the Mach number field observed on Figures 16-17 and analogous plots for the other five points omitted (for the sake of brevity). For points 1, 4, 6, 7, the upper-surface shock is pushed downstream thus enlarging the suction zone, and by this effect, increasing the lift. The effect on the lower-surface shock is opposite. Indeed, $\lambda_{1}$ is positive at these point. For points 2, 3 and 5 , the upper-surface shock is pushed upstream and the lower-surface shock is shifted upstream. The main effect is caused by the upper-surface shock displacement and the lift is decreased. Detailed plots of Mach number change due to residual perturbation is presented in Figures 16-17 for points 5 and 7 . When now the perturbation is made at point 5 , the Mach number locally altered along an oblique line impinging the shock-wave at the wall. Lastly, when the perturbation is made at point 7 , the wave is reflected on the upper surface, and impinges the shock-wave, creating a zone of strong influence. 


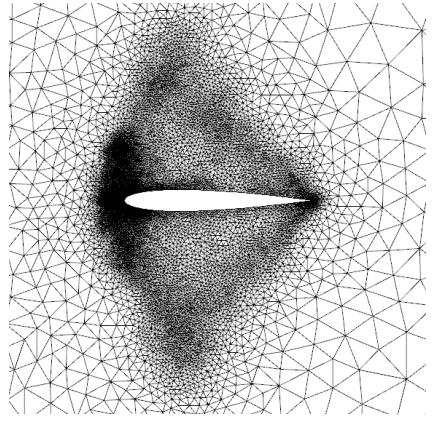

Figure 18: Supersonic conditions. Error-based method [47]

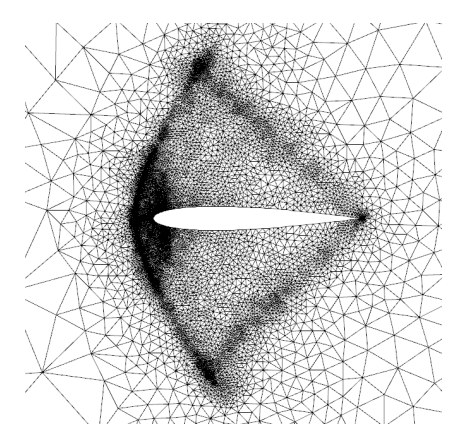

Figure 19: Supersonic conditions, $\theta$ based

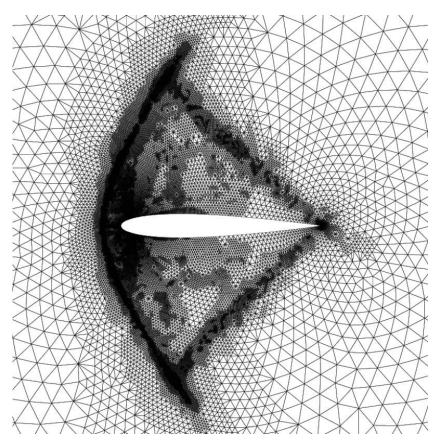

Figure 20: Supersonic conditions, dissipation-based [14]
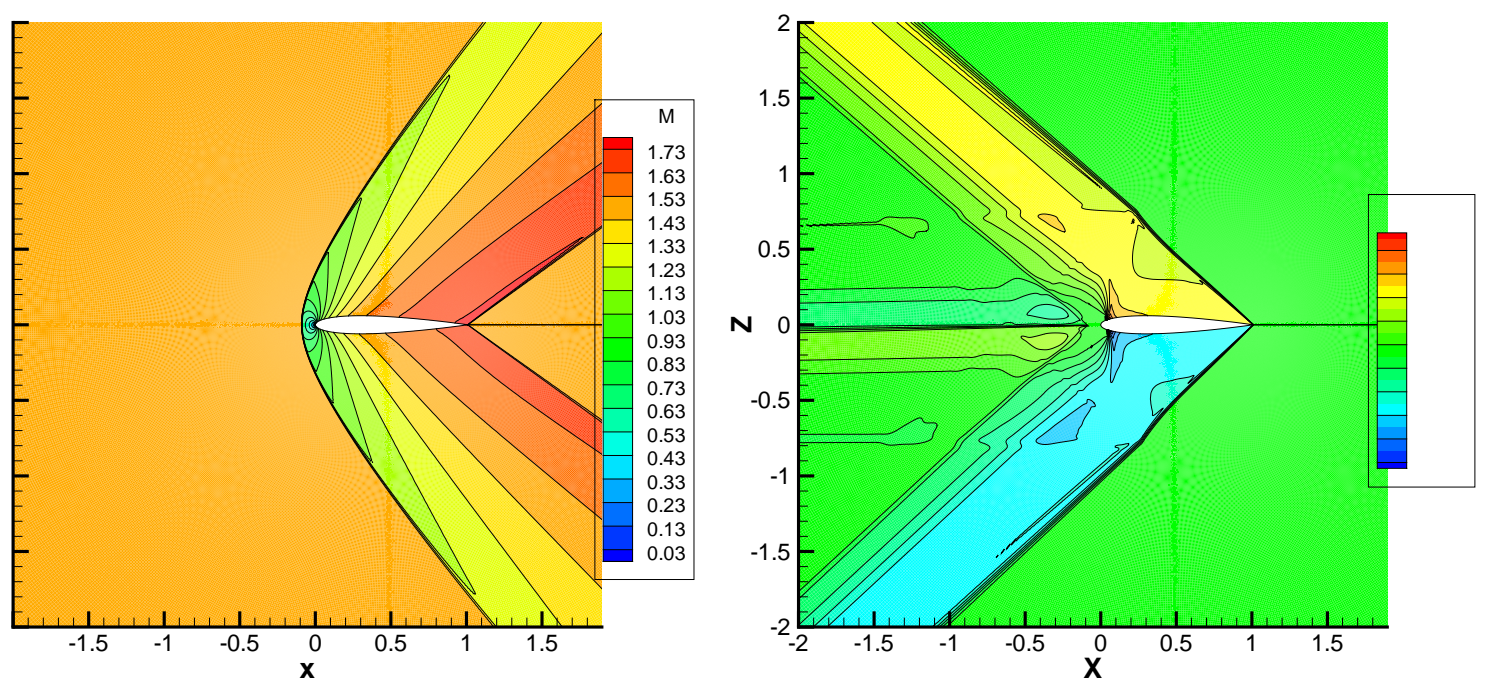

Figure 21: Supersonic case $\S 5.3$ iso-Mach number lines (left) $\Delta M=1.5$, iso-lines of first-component of lift adjoint vector (right)

In the supersonic case (Figures 18 to 20), a detached shock-wave is formed ahead of the body, and the supersonic flow is uniformly constant upstream of it. Thus, the sole upstream influence is concentrated in the shock geometry information, a zone of evident mesh concentration requirement. In particular, the fine mesh requirement of the stagnation-point streamline disappears upstream the shock. The subsonic bubble about the stagnation point and a small zone downstream close to the profile, upper and lower side, are regions of very high mesh density. The secondary shock is a fish-tail shock-wave; its base is at the trailing edge. Here, the region downstream the profile is supersonic and has no upstream influence. More precisely, the adjoint fields of the lift (first component figure 21), describes the geometric zone of flow influence for the lift (and actually the other near field functions functions) : a large angular section based on the trailing edge has no influence on lift (and other near field function values). Conversely, three zones based on the profile and directed upwind with polar angle $139,2^{\circ} 181^{\circ}$ and $-137,2^{\circ}$ are the zones of significant influence. This is of course fully consistent with the theory of simple waves for supersonic flows applied to adjoint equation and recalled in annex A with assumed constant flow defined by the far-field conditions $\left(\mathrm{M}_{\infty}, \mathrm{AoA}\right)$ - the values of angles $\theta$ and $\alpha$ in Annex $\mathrm{A}$ being the equal to $\theta=\mathrm{AoA}=1^{\circ} \quad \alpha=\sin ^{-1}\left(1 / \mathrm{M}_{\infty}\right) \simeq 41,8^{\circ}$. Consequently, two discontinuous lines for adjoint fields arise from trailing edge ; these are lines separating characteristics of the same family starting at the airfoil wall where adjoint field is non zero or from the far-field boundary downstream where the corresponding adjoint characteristic variable is zero. It is then interesting to note that the vicinity of these discontinuous lines is more refined that the region between this line and the profile where the adjoint has higher values but lower gradient. This should be linked with the various expressions of the error in computable correction - [47] equations (11) (12) (14) - that also indicates that zones of hight adjoint gradient should also be 
refined.

\section{Conclusion}

The more-widely usage of adjoint formulations has led several authors to reconsider mesh-adaptation strategies. In particular, the Venditti-Darmofal approach [47] has become a major reference in finitevolumes computational aerodynamics for goal-oriented mesh adaptation. In their method, a background fine mesh is used over which the flow field and the adjoint vector are extrapolated to identify precisely where it is adequate to densify a coarse mesh in order to achieve best accuracy on the function of interest. Presently, we have proposed to employ an adjoint-based sensor calculated over the current grid to identify these critical zones, thus eluding the necessity of a finer-grid estimation and gaining in simplicity and memory storage.

Eulerian-flow simulation tests were made using the ONERA finite-volume code elsA [7] in the unstructuredgrid mode. Flow fields over a classical airfoil in subsonic, transonic and supersonic regimes were calculated and analyzed. For this type of flows, lift or drag-oriented mesh adaptation is active unsurprisingly close to shock-waves, but also where the adjoint varies rapidly. The latter areas include the zone upwind the support of the function of interest and, in the supersonic case, the boundary of the dependence zones defined by the function support and the directions given by simple wave theory. They also include less intuitive zones like the hat-shaped adjoint isolines observed in the transonic test-case (figure 21). This was clarified using the identification of the adjoint vector as the sensitivity of the function of interest with respect to a change in the residual [20]: these non-intuitive strong value/strong gradient adjoint areas appeared as zones where a change in the scheme residual is propagated - with possible reflections on solid boundaries - affecting shock locations and, consequently, lift and drag values.

Besides, our adapted meshes were compared with those obtained by the Venditti and Darmofal method, and found to be very similar, thus accrediting our method in this context. In both method, the zones to be densified reflect the influence of both state and adjoint vectors (equation (12) for reference method [47] ; equations (2) and (3) for proposed method)

Although efforts were mostly directed towards airfoil computations, it should be pointed out that our sensor was also successfully tested with structured grids in the context of three-dimensional Euler and (RANS) flow over various configurations [33], and found adequate to qualify large-size meshes for complex applications. Anisotropic mesh refinement was up to now used only marginally with this sensitivity to nodes displacements method: in [5] the sensor has been used to define the number of mesh points in a boundary layer whereas the local structure of the mesh and the law of successive width are defined a priori. More sophisticated extension to anisotropic adaptation may mimic the method of Venditti and Darmofal for 2D viscous flows [48] where cell-size in driven by their classical criterion and cell aspect is defined from the Hessian of a scalar field.

\section{Acknowledgements}

The authors would like to warmly thank A. Jameson and JC. Vassberg for allowing the co-workers of D. Destarac to use the meshes of their well-known grid-convergence study [44, 45].

\section{A Local linear waves analysis for Euler continuous adjoint equa- tion}

In this appendix, the continuous form of the adjoint equation associated with the steady two-dimensional Euler equations is examined to identify the directions along which information travels locally in the flow. A computational domain $\Omega$ about an airfoil is considered. Its boundary $\Gamma$ is split in far field $\Gamma_{f f}$ and wall $\Gamma_{w}$. The outward normal vector is denoted $n$. The adjoint equation inside $\Omega$ results from the transposition of the linearized form of the Euler equations [2][24] :

$$
-A^{t} \frac{\partial \lambda}{\partial x}-B^{t} \frac{\partial \lambda}{\partial y}=0 \quad \text { in } \Omega
$$


where $A$ and $B$ are the usual Jacobian matrices, and the superscript ${ }^{t}$ indicates transposition. At a boundary point, $A n_{x}+B n_{y}=P D P^{-1}$ where $D$ is diagonal. Hence the column of $P$ are the right eigenvectors of $A n_{x}+B n_{y},\left(P^{-1} W\right)$ are the characteristic variables at the boundary, $\left(P^{T} \lambda\right)$ are the characteristic adjoint variables at the boundary [24]. The boundary conditions for drag adjoint are

$$
\begin{array}{cc}
n_{x} \lambda_{2}+n_{y} \lambda_{3}+\frac{2}{\rho_{\infty} V_{\infty}^{2}}\left(n_{x} \cos (A o A)+n_{y} \sin (A o A)\right)=0 & \text { on } \Gamma_{w}, \\
\left(P^{t} \lambda\right)_{k}=0 \text { if } D_{k k}>0 \text { (outgoing characteristic) } & \text { on } \Gamma_{f f} .
\end{array}
$$

If $D_{k k}<0$ (incoming characteristic), the direct characteristic variable $\left(P^{-1} W\right)_{k}$ is fixed and the corresponding $\left(P^{T} \lambda\right)_{k}$ adjoint characteristic variable is unconstrained. For lift-adjoint the boundary condition on $\Gamma_{w}$ gets

$$
n_{x} \lambda_{2}+n_{y} \lambda_{3}+\frac{2}{\rho_{\infty} V_{\infty}^{2}}\left(-n_{x} \sin (A o A)+n_{y} \cos (A o A)\right)=0 \quad \text { on } \Gamma_{w} .
$$

The privileged directions of flow information are given locally by the simple waves associated with equation (10). Such solutions are of the form

$$
\lambda(x, y)=\phi(x \sin \gamma-y \cos \gamma) \lambda_{0}
$$

where $\gamma$ is the angle made by the direction of propagation with the $x$-axis, the vector $\lambda_{0}$ represents the convected information. and $\phi$ is a scalar function modulating the magnitude of the $\lambda$ in the direction normal to convection Injecting this in 10 gives:

$$
\phi^{\prime}(x \sin \gamma-y \cos \gamma) \times\left(\sin \gamma A^{t}-\cos \gamma B^{t}\right) \lambda_{0}=0
$$

This equation admits a non-trivial solution $\lambda_{0}$ if and only if

$$
\operatorname{det}(\sin \gamma A-\cos \gamma B)^{t}=0 .
$$

This characteristic equation is the same as for the linearized Euler equations themselves since transposition plays no role in it. The eigenvalues of the matrix $(\sin \gamma A-\cos \gamma B)^{t}$ are well known : if $u=V \cos \beta$ and $v=V \sin \beta$ are the velocity components, and $c$ is the speed of sound, these eigenvalues are : $u \sin \gamma-v \cos \gamma$ (double) and $u \sin \gamma-v \cos \gamma \pm c$ (both simple). Hence $\gamma$ is the solution of one of the equations :

$$
u \sin \gamma-v \cos \gamma=0, \quad u \sin \gamma-v \cos \gamma \pm c=0 .
$$

The first equation gives

$$
\sin (\gamma-\beta)=0 \quad \text { that is: } \gamma=\beta \text { or } \gamma=\beta+\pi
$$

and this corresponds to the two opposite semi-axes supported by the velocity vector, corresponding to information travelling downwind by material transport, or information emanating from upwind accordingly. The second equation in (11) admits solutions only if the flow is locally supersonic. Then, denoting $\alpha=\sin ^{-1}(1 / M)$ the Mach angle, it writes:

$$
\sin (\gamma-\beta) \pm \sin \alpha=0
$$

and it yields:

$$
\gamma=\beta-\alpha, \quad \gamma=\beta+\pi+\alpha \quad \text { or } \quad \gamma=\beta+\alpha, \quad \gamma=\beta+\pi-\alpha .
$$

These equations define the four semi-axes bounding the Mach cone. Evidently, the domains of influence/dependence are inverse one another for state and adjoint variables. Hence, for the adjoint variables, information propagates along the direction $\gamma=\beta+\pi \pm \alpha$ and backwards along streamlines. 
B Evolution of mesh density during adaptation for lifting transonic and supersonic test-cases

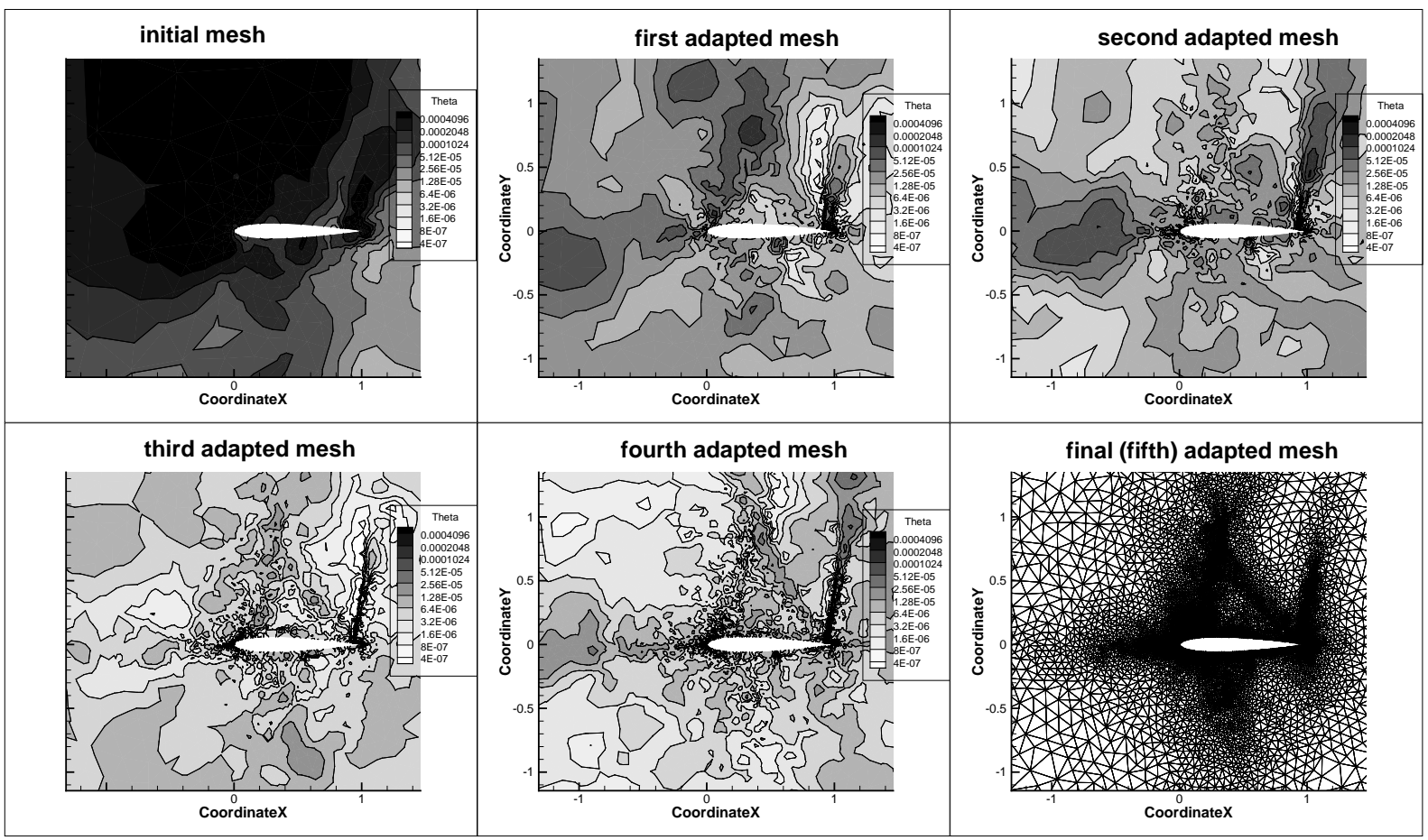

Figure 22: Fields of $\theta$ indicator at the successive steps. Lift-oriented mesh adaptation for the first transonic test-case $\left(\mathrm{M}_{\infty}=0.85 \mathrm{AoA}=2^{\circ}\right)$

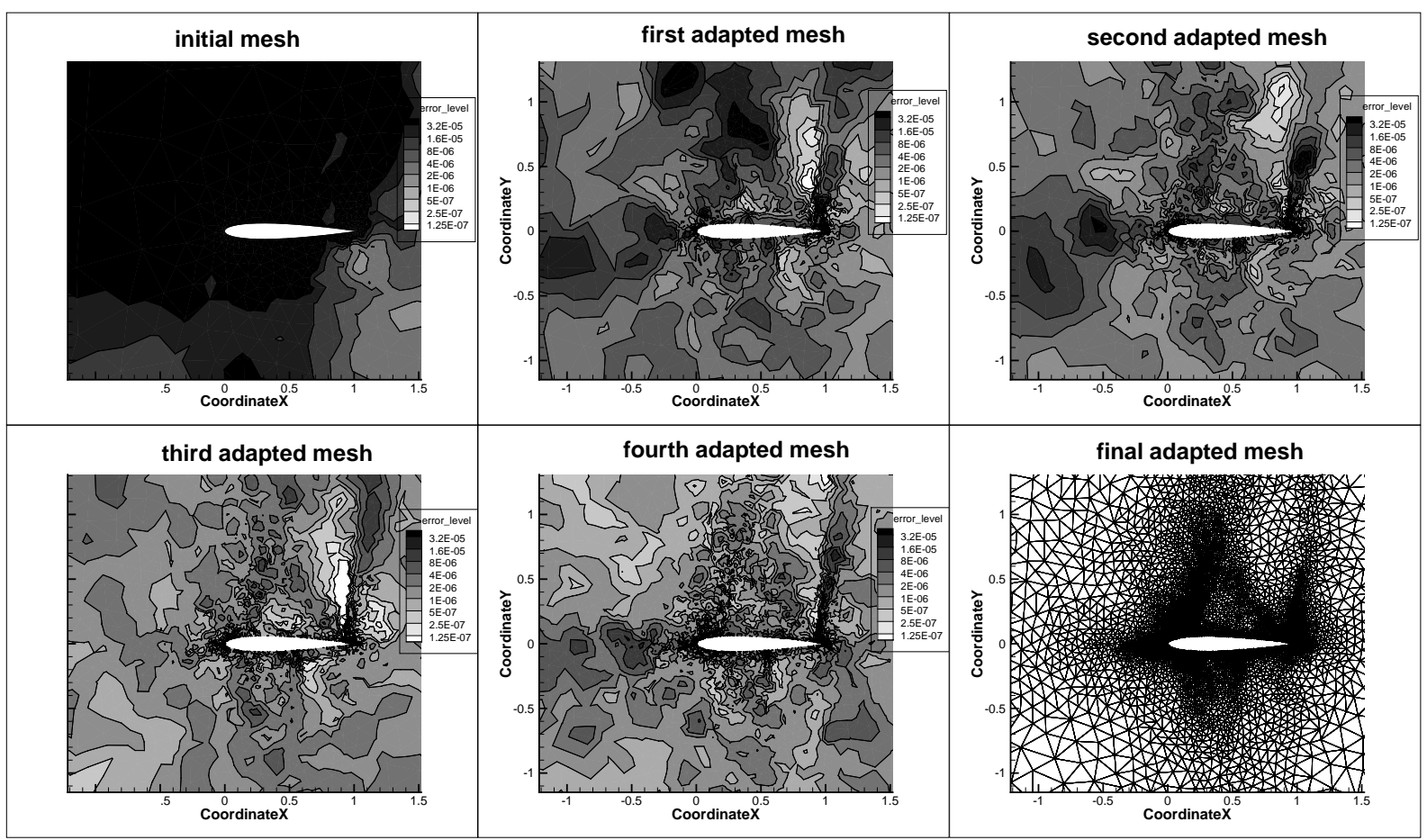

Figure 23: Fields of absolute value of local contribution to error in computable correction of reference method at the successive steps. Lift-oriented mesh adaptation for the first transonic test-case $\left(\mathrm{M}_{\infty}=0.85\right.$ $\mathrm{AoA}=2^{\circ}$ ) 


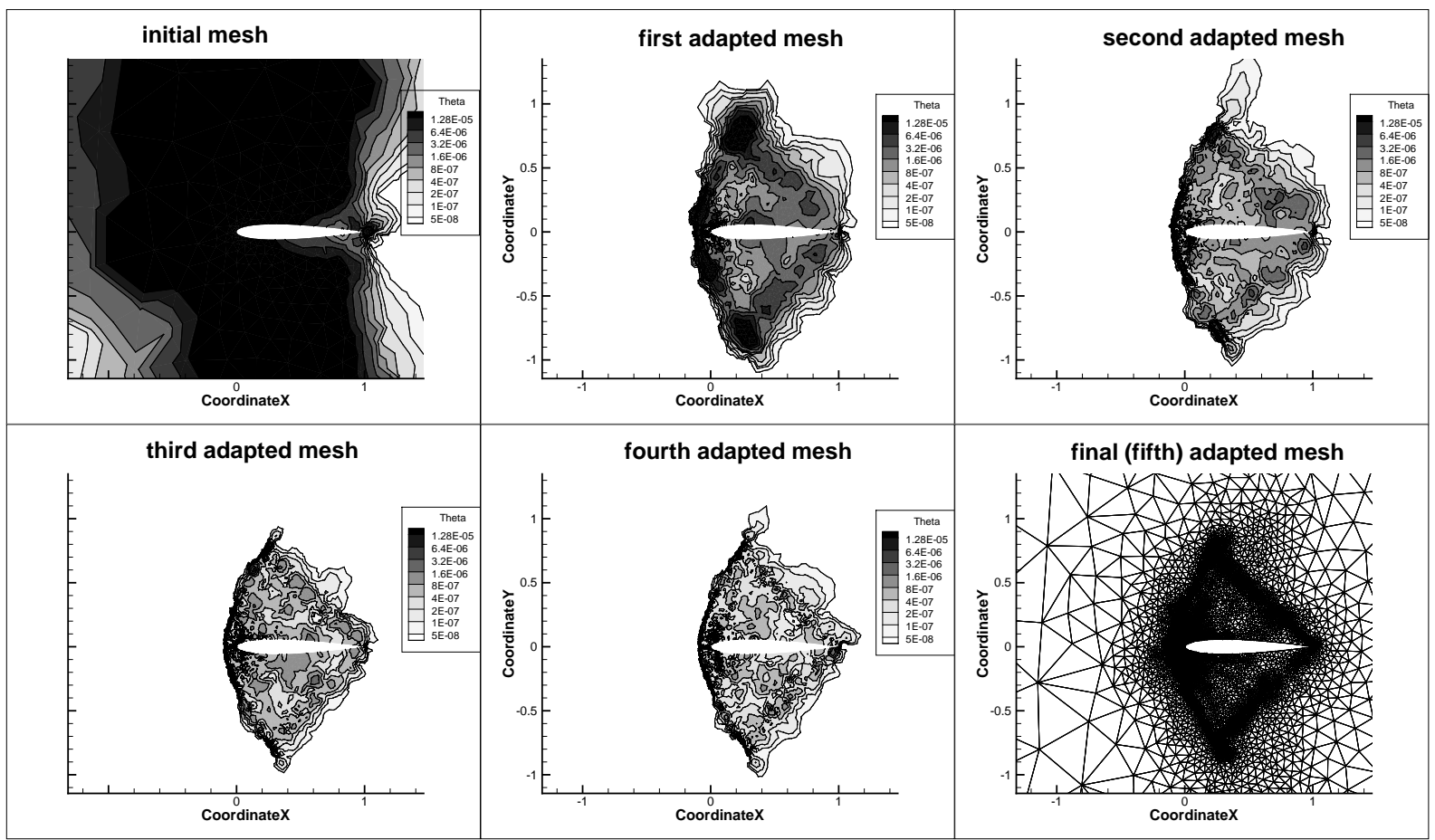

Figure 24: Fields of $\theta$ indicator at the successive steps. Lift-oriented mesh adaptation for the supersonic test-case $\left(\mathrm{M}_{\infty}=1.5 \mathrm{AoA}=1^{\circ}\right)$

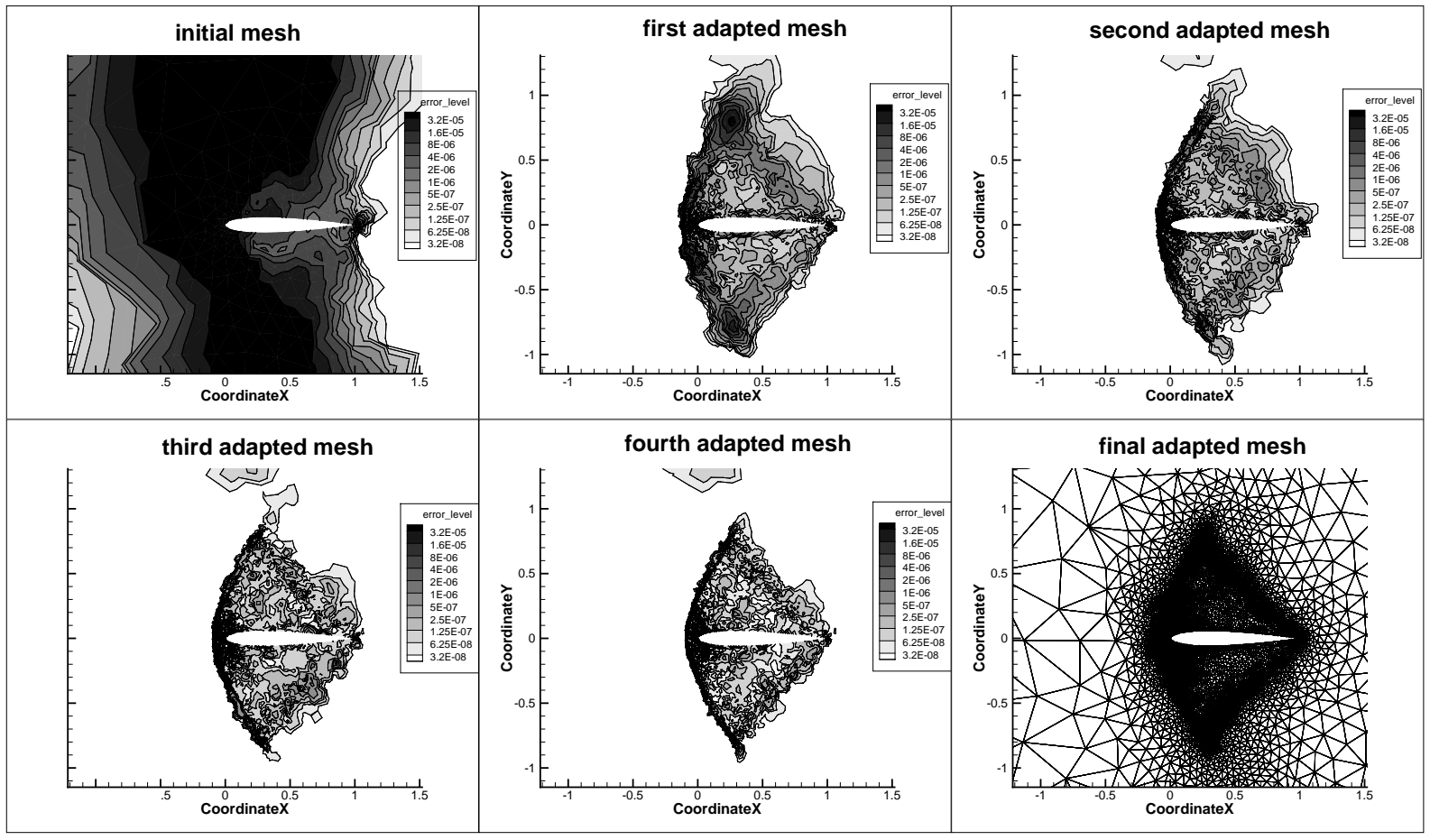

Figure 25: Fields of absolute value of local contribution to error in computable correction of reference method at the successive steps. Lift-oriented mesh adaptation for the supersonic test-case $\left(\mathrm{M}_{\infty}=1.5\right.$ $\left.\mathrm{AoA}=1^{\circ}\right)$

\section{References}

[1] G.D. van Albada, B. van Leer W.W. Roberts Jr. A comparative study of computational methods in cosmic gas dynamics. Astronomy and Astrophysics. 1008 (1982) 76-84. 
[2] W.K. Anderson, V. Venkatakrishnan. Aerodynamic design optimization on unstructured grids with a continuous adjoint formulation. Computers and Fluids. 28 (1999) 443-480.

[3] T.J. Baker. Mesh adaptation strategies for problems in fluid dynamics. Finite Elements in analysis and Design. 25 (1997) 243-273.

[4] R. Biswas, R Strawn. Tetrahedral and hexaedral mesh adaptation for CFD computations. Applied Numerical Mathematics. 26 (1998) 131-151.

[5] S. Bourasseau. Contribution à une méthode de raffinement de maillage basée sur le vecteur adjoint pour le calcul de fonctions aérodynamiques. Thèse de l'Université de Nice-Sophia Antipolis. 2015.

[6] R. Boussetta, T. Coupez, L. Fourment. Adaptive remeshing based on a posteriori error estimation for forging simulation. Computer Methods in Applied Mechanics and Engineering. 195 (2006) 6626-6645.

[7] L. Cambier, S. Heib, S. Plot. The Onera elsA CFD software : input from research and feedback from industry, Mechanics \& Industry. 14(3) (2013) 159-174.

[8] T. Coupez. Metric construction by length distribution tensor and edge based error for anisotropic adaptive meshing. Journal of Computational Physics 230 (2011) 2391-2405.

[9] D. Destarac, Far-Field / Near-Field Drag Balance and Applications of Drag Extraction in CFD, VKI Lecture Series 2003, CFD-based Aircraft Drag Prediction and Reduction, Rhode Saint Genèse (Belgium), February 3-7, 2003, National Institute of Aerospace, Hampton (VA), November 3-7, 2003.

[10] B. Diskin, N.K. Yamaleev. Grid Adaptation using Adjoint-Based Error Minimization. In AIAA Paper series: Paper 2011-3986. 2011.

[11] C. Dobrzynski. MMG3D. User guide. INRIA Technical Report 422. 2012.

[12] R.P. Dwight. Efficient A Posteriori Error Estimation for Finite Volume Methods. NATO Research and Technology Organisation, Proceedings of AVT-147 on Computational Uncertainty, Athens, 2007.

[13] R.P. Dwight. Goal-oriented mesh adaptation using a dissipation based error indicator. International Journal for Numerical Methods in Fluids. 56(8) (2007) 1193-2000.

[14] R.P. Dwight. Heuristic a posteriori estimation of error due to dissipation in finite volume schemes and application to mesh adaptation. Journal of Computational Physics. 227 (2008) 2845-2863.

[15] P.J. Frey, F. Alauzet. Anisotropic mesh adaptation for CFD computations. Computer Methods in Applied Mechanics and Engineering. 194 (2005) 5068-5082.

[16] D. Gueguan, O. Allain, A. Dervieux, F. Alauzet. An $\mathrm{L}^{\infty} \mathrm{L}^{p}$ mesh adaptive method for computing unsteady bi-fluid flows. International Journal for Numerical Methods in Fluids. 84 (2010) 1376-1406.

[17] A. Jameson, W. Schmidt, E. Turkel. Numerical Solutions of the Euler Equations by Finite Volume Methods Using Runge-Kutta Time-Stepping Schemes. In AIAA Paper series: Paper 81-1259. 1981.

[18] A. Jameson. Aerodynamic design by control theory. Journal of Scientific Computing. 3(3) (1988) $233-260$.

[19] K.J. Fidkowski, P.L. Roe. An entropy adjoint approach to mesh refinement. SIAM Journal of Scientific Computing. 32(3) (2010) 1261-1287.

[20] K.J. Fidkowski, D.L. Darmofal. Output-Based Error Estimation and Mesh Adaptation in Computational Fluid Dynamics: Overview and Recent Results. AIAA Journal. 49(4) (2011) 673-694.

[21] K.J. Fidkowski, M.A. Ceze, P.L. Roe. Entropy-Based Drag Error Estimation and Mesh Adaptation in Two Dimensions. Journal of Aircraft. 49(5) (2012) 1485-1496.

[22] F. Fraysse, J. de Vincente, E. Valero. The estimation of truncation error by $\tau$-estimation revisited. Journal of Computational Physics. 231 (2012) 2457-3482.

[23] F. Fraysse, E. Valero, J. Ponsin. Comparison of Mesh Adaptation Using the Adjoint Methodology and Truncation Error Estimates. AIAA Journal. 50(9) (2005) 1920-1931. 
[24] M.B. Giles, N.A. Pierce. Adjoint equations in CFD: duality, boudary conditions, and solution behaviour. In: AIAA paper series: Paper 1997-1850. 1997.

[25] W.G. Habashi, J. Dompierre, Y. Bourgault, M. Fortin, M.-G. Vallet. Certifiable Computational Fluid Dynamics Through Mesh Optimization. AIAA Journal. 36(5) (1998) 703-711.

[26] R. Hartmann, P. Houston. Adaptive discontinuous finite element methods for the compressible Euler equations. Journal of Computational Physics. 183 (2002) 508-532.

[27] R. Hartmann. Adaptative discontinuous Galerkin methods with shock-capturing for the compressible Navier-Stokes equations. International Journal for Numerical Methods in Fluids. 51 (2006) 11311156.

[28] R. Hartmann, J. Held, T. Leicht. Adjoint-based error estimation and adaptative mesh refinement for the RANS and k- $\omega$ turbulence model equations. Journal of Computational Physics. 230 (2011) 4268-4284.

[29] G. Jannoun, E. Hachem, J. Veysset, T. Coupez. Anisotropic meshes with time-stepping control for unsteady convection-dominated problems. Applied Mathematical Modelling. 39(7) (2015) 1899-1916.

[30] J. Peter, M. Nguyen-Dinh, P. Trontin. Goal oriented mesh adaptation using total derivative of aerodynamic functions with respect to mesh coordinates. With application to Euler flows. Computers and Fluids. 66 (2012) 194-214.

[31] A. Loseille, A. Dervieux, F.Alauzet. Fully anusotropic goal-oriented mesh adaptation for 3D steady Euler equations. Journal of Computational Physics. 229 (2010) 2866-2897.

[32] M. Nguyen-Dinh, J. Peter, R. Sauvage, M. Meaux, J.A. Désidéri. Mesh quality assessment based on aerodynamic functional output total derivatives. European journal of Mechanics B/Fluids. 45 (2014) $51-71$.

[33] M. Nguyen-Dinh. Qualification des simulations numériques par adaptation anisotropique de maillages. Thèse de l'Université de Nice-Sophia Antipolis. 2014.

[34] E. Nielsen, M. Park. Using an adjoint approach to eliminate mesh sensitivities in aerodynamic design. AIAA Journal. 44(5) (2005) 948-53.

[35] J. Peraire, M. Vahdati, K. Morgan, O.C. Zienkiewicz. Adaptive remeshing for compressible flow computations. Journal of Computational Physics. 72 (1987) 449-466.

[36] J. Peraire, J. Peiro, K. Morgan. Adaptive remeshing for three-dimensional compressible flow computations. Journal of Computational Physics. 103 (1992) 269-285.

[37] N.A. Pierce, M.B. Giles. Adjoint recovery of superconvergent functionals for PDE approximations. SIAM Review. 42 (2000) 247-264.

[38] N.A. Pierce, M.B. Giles. Adjoint and defect error bounding and correction for functional estimates. In: AIAA paper series, Paper 2003-3846. 2003.

[39] P.J. Roache. Perspective: A Method for Uniform Reporting of Grid Refinement Studies. Journal of Fluids Engineering. 116(3) (1994) 405-413.

[40] P.L. Roe. Approximate Riemann solvers, parameter vectors, and difference schemes. Journal of Computational Physics. 43 (1981) 357-372.

[41] P.L. Roe. Characteristic-based schemes for the Euler equations. Annual Review of Fluid Mechanics. 18 (1986) 337-365.

[42] C.L. Rumsey, J.L. Thomas. Application of FUN3D and CFL3D to the Third Workshop on CFD Uncertainty Analysis. Proceedings of the Third Workshop on CFD Uncertainty Analysis. Lisbon. 2008.

[43] G. Todarello. Goal oriented adaptation of unstructured meshes. Application to finite volume methods. MSc Thesis. TU-Delft. 2014. 
[44] J.C. Vassberg, A. Jameson. In pursuit of grid convergence, part I: two-dimensional Euler solution. In: AIAA paper series, Paper 2009-4114. 2009.

[45] J.C. Vassberg, A. Jameson. In pursuit of grid convergence for Two-Dimensional Euler Solutions, Journal of Aircraft. 47 (2010) 1152-1166.

[46] D.A. Venditti, D.L. Darmofal. Adjoint error estimation and grid adaptation for functional outputs: Application to quasi-one-dimensional flow. Journal of Computational Physics. 164 (2000) 40-69.

[47] D.A. Venditti, D.L. Darmofal. Grid adaptation for functional outputs: Aplication to two-dimensional inviscid flows. Journal of Computational Physics. 176 (2002) 40-69.

[48] D.A. Venditti, D.L. Darmofal. Anisotropic grid adaptation for functional outputs: Application to two-dimensional viscous flows. Journal of Computational Physics. 187 (2003) 22-46.

[49] G. Warren, W. Anderson, J. Thomas, S. Krist. Grid convergence for adaptive methods. In AIAA Paper series: Paper 1991-1592. 1991.

[50] M. Widhalm, J. Brezillon, T. Leicht. Investigation of adjoint based gradient computation for realistic $3 \mathrm{~d}$ aero-optimization. Proceedings of 13th AIAA/ISSMO Multidisciplinary Analysis Optimization Conference. 2010.

[51] N.K. Yamalev, B. Diskin, K. Pathak. Error Minimization via Adjoint-Based Anisotropic Grid Adaptation In AIAA Paper series: Paper 2010-4436. 2010.

[52] M. Yano, D.L. Darmofal. An optimization based framework for anisotropic simplex mesh adaptation. Journal of Computational Physics. 231 (2012) 7626-7649. 